\title{
SUSY breaking in local string/F-theory models
}

\author{
R. Blumenhagen, ${ }^{a}$ J.P. Conlon, ${ }^{b}$ S. Krippendorf, ${ }^{c}$ S. Moster ${ }^{a}$ and F. Quevedo ${ }^{c, d}$ \\ ${ }^{a}$ Max-Planck-Institut für Physik, \\ Föhringer Ring 6, 80805 München, Germany \\ ${ }^{b}$ Rudolf Peierls Centre for Theoretical Physics, \\ 1 Keble Road, Oxford OX1 3NP, U.K. \\ ${ }^{c}$ DAMTP, University of Cambridge, \\ Wilberforce Road, Cambridge, CB3 0WA, U.K. \\ ${ }^{d}$ CERN PH-TH, \\ CH 1211, Geneva 23, Switzerland \\ E-mail: blumenha@mppmu.mpg.dex, j.conlon1@physics.ox.ac.uk, \\ s.krippendorf@damtp.cam.ac.uk, moster@mppmu.mpg.de, \\ f.quevedo@damtp.cam.ac.uk
}

ABSTRACT: We investigate bulk moduli stabilisation and supersymmetry breaking in local string/F-theory models where the Standard Model is supported on a del Pezzo surface or singularity. Computing the gravity mediated soft terms on the Standard Model brane induced by bulk supersymmetry breaking in the LARGE volume scenario, we explicitly find suppressions by $M_{s} / M_{P} \sim \mathcal{V}^{-1 / 2}$ compared to $M_{3 / 2}$. This gives rise to several phenomenological scenarios, depending on the strength of perturbative corrections to the effective action and the source of de Sitter lifting, in which the soft terms are suppressed by at least $M_{P} / \mathcal{V}^{3 / 2}$ and may be as small as $M_{P} / \mathcal{V}^{2}$. Since the gravitino mass is of order $M_{3 / 2} \sim M_{P} / \mathcal{V}$, for TeV soft terms all these scenarios give a very heavy gravitino $\left(M_{3 / 2} \geq 10^{8} \mathrm{GeV}\right)$ and generically the lightest moduli field is also heavy enough $(m \geq 10 \mathrm{TeV})$ to avoid the cosmological moduli problem. For TeV soft terms, these scenarios predict a minimal value of the volume to be $\mathcal{V} \sim 10^{6-7}$ in string units, which would give a unification scale of order $M_{\mathrm{GUT}} \sim M_{s} \mathcal{V}^{1 / 6} \sim 10^{16} \mathrm{GeV}$. The strong suppression of gravity mediated soft terms could also possibly allow a scenario of dominant gauge mediation in the visible sector but with a very heavy gravitino $M_{3 / 2}>1 \mathrm{TeV}$.

KEYwords: Flux compactifications, F-Theory, Intersecting branes models

ArXiv EPrint: 0906.3297 


\section{Contents}

1 Introduction 1

2 Effective field theories and moduli stabilisation 3

2.1 Gauge couplings on the GUT brane 4

2.2 Moduli stabilisation 5

2.2.1 Fixing the non-standard model/GUT cycles 6

$\begin{array}{lll}\text { 2.2.2 } & \text { Fixing the standard model/GUT cycle } & 7\end{array}$

2.3 Including matter fields 8

2.4 Summary of EFTs 9

3 Gravity mediated soft terms $\quad 9$

$\begin{array}{lll}3.1 & \text { Structure of soft terms } & 10\end{array}$

$\begin{array}{lll}3.1 .1 & \text { Gaugino masses } & 10\end{array}$

$\begin{array}{lll}3.1 .2 & \text { Squark/slepton masses } & 11\end{array}$

$\begin{array}{lll}3.1 .3 \hat{\mu} / \hat{\mu} B \text {-terms } & 12\end{array}$

$\begin{array}{lll}3.1 .4 & \text { A-terms } & 12\end{array}$

3.1.5 Anomaly mediated gaugino masses 13

$\begin{array}{lll}3.2 & \text { Summary of gravity mediated soft masses } & 13\end{array}$

$\begin{array}{lll}3.3 & \text { Uplift and cancellations } & 14\end{array}$

4 Consequences for supersymmetry breaking $\quad 16$

$\begin{array}{lll}4.1 & \text { Gauge mediated scenarios } & 17\end{array}$

$\begin{array}{lll}4.2 & \text { Implications for the cosmological moduli problem } & 19\end{array}$

5 Conclusions $\quad 20$

$\begin{array}{ll}\text { A F-Terms } & 23\end{array}$

$\begin{array}{ll}\text { B Vanishing D-terms including matter } & 25\end{array}$

\section{Introduction}

During the last years moduli stabilisation, in particular for Type IIB orientifolds on compact Calabi-Yau threefolds, has been under intense study and several scenarios have been proposed. The original example is the KKLT scenario [1], where dilaton and complex structure moduli are fixed at tree-level by fluxes while Kähler moduli are stabilised via instanton generated terms in the superpotential

$$
W=W_{0}+A e^{-a T_{s}} .
$$


As a generalisation of this, including also next-to-leading order corrections to the Kähler potential, the volume of the compactification manifold $\mathcal{V}$ can be stabilised at exponentially large values $[2,3]$. These large volume minima are quite generic [4] and exist whenever there is a four-cycle in the Calabi-Yau threefold which is shrinkable to zero size, such that the total volume of the space remains finite. These supersymmetry breaking Type IIB vacua have been called the LARGE Volume Scenario (LVS). Their phenomenological features were studied in very much detail for the string scale in the intermediate regime $M_{s} \simeq$ $10^{11} \mathrm{GeV}[5,6]$ leading to $\mathrm{TeV}$ soft terms and intermediate scales for the neutrino and axion sector of the MSSM in the preferred range. For computing the high scale soft terms it was assumed that the D7-branes supporting the MSSM gauge and matter fields wrap the same four-cycle supporting also the D3-brane instanton. In [7], it was pointed out that instanton zero mode counting actually forbids such a scenario and that the D7-branes and instantonic D3-branes should better wrap distinct four-cycles in the underlying Calabi-Yau manifold. In fact the Kähler moduli associated to the sizes of the four-cycles wrapped by the D7-branes can be stabilised by D-terms, often at string scale size at the boundary of the Kähler cone. In this sense the MSSM branes are sequestered from the bulk of the Calabi-Yau.

A way of realising the MSSM gauge and matter fields in the LVS is by studying fractional D3-branes at the singular point, discussed in [8, 9]. Various low-energy models were studied on the first two del Pezzo surfaces $d P_{0}$ and $d P_{1}$, allowing for both GUTlike and extended MSSM scenarios. From the effective field theory point of view, both scenarios are similar since after stabilising the moduli, both vacua are at or close to the singular point.

A very similar scenario was proposed recently in the context of local F-theory models with an SU(5) GUT brane. First of all, it was realised that some of the model building problems one had with realising simple GUT groups in orientifold constructions [10] are nicely reconciled in F-theory models on elliptically fibered Calabi-Yau fourfolds [11-18]. The reason for this substantial improvement is that F-theory is genuinely non-perturbative and also allows for the appearance of exceptional groups $E_{6}, E_{7}, E_{8}$, which are supported along a complex surface in the base threefold, over which the elliptic fiber degenerates appropriately. ${ }^{1}$ As a consequence, by a further breaking also the spinor representation of an SO(10) GUT and the top-quark Yukawa couplings $10105_{H}$ in SU(5) GUTs can be realised.

Moreover, it was proposed in [13] that such models can allow for an essentially local treatment, if there exists a limit in which gravity decouples from the gauge theory on the GUT brane. Geometrically this means that the space transverse to the brane can become arbitrary large or from a different perspective that the four-cycle the brane is wrapping can shrink to zero size. Such four-cycles are so-called del Pezzo surfaces, which are $\mathbb{P}^{2}$ blown up at up to eight points. Since there exists a limit where gravity decouples from the physics on the $\mathrm{SU}(5)$ brane, one expects that for gravity induced couplings on the

\footnotetext{
${ }^{1}$ In the Type IIB interpretation, such loci support general $(p, q)$ seven brane systems, where the extra states are realised by massless string junctions.
} 
brane there exists an $M_{s} / M_{P}$ suppression relative to their general values. This decoupling argument was used heavily when studying the further phenomenological implications of local F-theory GUTs $[19,20]$. In particular, it was argued that, since gravity/moduli mediated supersymmetry breaking soft terms on the GUT brane should also experience such a suppression, gauge mediation could become the dominant source. Indeed, under this assumption a very nice numerology for the soft terms was deduced, which besides flavour universality includes a solution to the $\mu / \mu B$ problem and a candidate for the QCD axion solving the strong CP-problem. The gauge mediation was parametrised in the usual way by the non-zero VEV of a scalar field $\langle X\rangle=x+\theta^{2} F$ mediated to the MSSM by charged messenger fields. Note that in this scenario the gravitino mass was assumed to be dominantly set by gauge mediation. Therefore, the gravitino was the LSP with a mass of $1 \mathrm{GeV}$.

Since both the LARGE volume scenario and local F-theory GUTs require the same kind of geometry, i. e. Calabi-Yau respectively base threefolds containing del Pezzo surfaces, it is natural to combine these two set-ups and study, for this concrete moduli stabilisation mechanism in the bulk, the computable effects of gravity mediation for the physics on the GUT brane. It is the primary aim of this paper, to compute for a minimal set-up these gravity mediated soft terms explicitly and compare them with the expectation of an $\mathcal{V}^{-\frac{1}{2}}=M_{s} / M_{P}$ suppression. Indeed, as we will show such a computation requires to compute the soft terms at next-to-leading orders in $1 / \mathcal{V}$.

This paper is organised as follows: In section 2 we review the geometric framework of local GUTs and the LARGE volume approach to moduli stabilisation that is applicable in this regime. In section 3 we describe the computation of gravity mediated soft terms. We describe how the soft terms cancel at $\mathcal{O}\left(M_{3 / 2}\right)$ and how it is necessary to consider sub-leading corrections. We find sub-leading contributions to soft terms at order $\mathcal{O}\left(M_{3 / 2} / \sqrt{\mathcal{V}}\right)=\mathcal{O}\left(\frac{M_{3 / 2}^{3 / 2}}{M_{P}^{1 / 2}}\right)$. In certain circumstances these contributions can also cancel and we give a set of well posed assumptions when this can occur. In section 4 we discuss the implications from these soft terms for both gauge mediation and the cosmological moduli problem, and in section 5 we conclude.

\section{Effective field theories and moduli stabilisation}

The minimal set-up we are investigating in this paper is that we have a threefold with at least three four-cycles, one large cycle and two small del Pezzo four-cycles, i. e. the threefold is of the (strong) swiss-cheese type. One of the del Pezzos supports the SU(5)/MSSM gauge theory while the other one can support a D3-brane instanton inducing a non-perturbative contribution to the superpotential. Therefore, for the size of the overall volume and the instanton four-cycle (without any further contributions) there exists the non-supersymmetric AdS-type LARGE volume minimum. Since the GUT brane is localised on a del Pezzo surface orthogonal to the instantonic del Pezzo and the size of the GUT brane is fixed by $\mathrm{D}$-terms at small values, the previous computations of the gravity induced soft terms 
should be modified. The same calculation is also necessary for the case that the GUT cycle is collapsed at the quiver locus.

There are two basic regimes where the effective field theory (EFT) for light modes is reliable:

- All of the 4-cycles, including the standard model or GUT cycle are larger than the string scale. This is the geometric regime.

- The size of the standard model cycle is much smaller than the string scale. It is a standard blow-up mode expanded around its vanishing value corresponding to the del Pezzo singularity. Fortunately string theory is under control at the singularity and the EFT can be safely defined in an expansion on the blow-up mode.

Since the D-term conditions tend to prefer a small value of the standard model cycle, it is important to understand the physics in both regimes of validity of EFT. It is clear that these are two different effective field theories for standard model physics. But, as we will see, since the standard model cycle does not participate in the breaking of supersymmetry, the structure of soft breaking terms will be the same in both cases.

Let us discuss the ingredients in some more detail.

\subsection{Gauge couplings on the GUT brane}

Let us recall the set-up for Type IIB respectively F-theory GUT models, where we use for concreteness the Type IIB orientifold language of [21, 22]. We consider the Type IIB string compactified on a compact Calabi-Yau threefold $\mathcal{M}$ modded out by an orientifold projection $\Omega \sigma(-1)^{F_{L}}$. The holomorphic involution is such that one gets O7- and O3planes. The base of the corresponding elliptically fibered four-fold is then $B_{3}=\mathcal{M} / \sigma$. The SU(5) GUT is localised on D7-branes wrapping a rigid del Pezzo surface $D_{a}$. The resulting tree-level $\mathrm{SU}(5)$ gauge kinetic function $f_{\mathrm{SU}(5)}=\frac{4 \pi}{g_{X}^{2}}+i \Theta$ is simply given by

$$
f_{\mathrm{SU}(5)}=T_{a}=\frac{1}{2 g_{s} \ell_{s}^{4}} \int_{D_{a}} J \wedge J+i \int_{D_{a}} C_{4}
$$

where $g_{s}=e^{\varphi}$ denotes the string coupling constant and $\operatorname{Vol}\left(D_{a}\right)=\frac{1}{2} \int_{D_{a}} J \wedge J$ is the volume of the del Pezzo surface $D_{a}$.

In orientifold models, we actually get on a stack of five D7-branes the Chan-Paton gauge group $\mathrm{U}(5)$, which allows for a non-vanishing gauge flux $\mathcal{F}_{a}$ in the diagonal $\mathrm{U}(1) \subset$ $\mathrm{U}(5)$. Since a del Pezzo is rigid and does not even contain any discrete Wilson lines, the gauge symmetry is broken to $\mathrm{SU}(3) \times \mathrm{SU}(2) \times \mathrm{U}(1)_{Y}$ by a non-trivial $\mathrm{U}(1)_{Y}$ gauge flux $\mathcal{F}_{Y}$ supported on a two-cycle $C_{a} \in H_{2}\left(D_{a}, \mathbb{Z}\right)$ which is trivial in $H_{2}(\mathcal{M}, \mathbb{Z})[13,14]$. As explained in [22], this way of breaking the $\mathrm{SU}(5)$ gauge group leads to a specific pattern of MSSM gauge couplings at the unification scale

$$
f_{i}=T_{a}-\frac{1}{2} \kappa_{i} S, \quad i \in\{1,2,3\}
$$


with

$$
\begin{aligned}
\kappa_{3} & =\int_{D_{a}} \mathcal{F}_{a}^{2}, \\
\kappa_{1} & =\int_{D_{a}} \mathcal{F}_{a}^{2}+\frac{3}{5}\left(\mathcal{F}_{Y}^{2}+2 \mathcal{F}_{a} \mathcal{F}_{Y}\right) .
\end{aligned}
$$

For concreteness we are using these orientifold relations in the following.

In the limit that the cycle is collapsed to the singularity, the gauge kinetic function takes a similar form:

$$
f_{i}=\delta_{i} S+s_{i k} T_{k},
$$

where now $T_{k}$ has to be understood as the blow-up modes that resolve the singularity. For $\mathbb{Z}_{n}$ singularities $\delta_{i}$ is universal; however for more complicated singularities $\delta_{i}$ can be non-universal. For applications to unification, we are interested in singularities where the different gauge groups have universal couplings at the singularity.

For both classes of local models the GUT unification scale and string scale differ significantly by a factor of the bulk radius. More precisely, the GUT unification scale $M_{X}$ is given by $M_{X}=R M_{s}$, where $R \sim \mathcal{V}^{1 / 6}$ is the bulk radius of the Calabi-Yau in string units. This can be seen through the Kaplunovsky-Louis relation between physical and holomorphic gauge couplings,

$$
\begin{aligned}
g_{a}^{-2}(\Phi, \bar{\Phi}, \mu)= & \operatorname{Re}\left(f_{a}(\Phi)\right)+\frac{\left(\sum_{r} n_{r} T_{a}(r)-3 T_{a}(G)\right)}{8 \pi^{2}} \ln \left(\frac{M_{P}}{\mu}\right)+\frac{T(G)}{8 \pi^{2}} \ln g_{a}^{-2}(\Phi, \bar{\Phi}, \mu) \\
& +\frac{\left(\sum_{r} n_{r} T_{a}(r)-T(G)\right)}{16 \pi^{2}} \hat{K}(\Phi, \bar{\Phi})-\sum_{r} \frac{T_{a}(r)}{8 \pi^{2}} \ln \operatorname{det} Z^{r}(\Phi, \bar{\Phi}, \mu) .
\end{aligned}
$$

Using the IIB Kähler potential $\hat{K}=-2 \ln \mathcal{V}$ and the behaviour for local models $\hat{Z}=\mathcal{V}^{-2 / 3}$ we obtain

$$
g_{a}^{-2}(\mu)-\frac{T(G)}{8 \pi^{2}} \ln g_{a}^{-2}(\mu)=\operatorname{Re}\left(f_{a}(\Phi)\right)+\beta_{a} \ln \left(\frac{\left(R M_{s}\right)^{2}}{\mu^{2}}\right),
$$

giving effective unification at $R M_{s}$. As described in [23-25], at the string level this dependence arises from the presence of tadpoles that are sourced in the local model but are only cancelled globally. This comes from the fact that the $\mathrm{U}(1)_{Y}$ flux that breaks the GUT group is on a two-cycle that is non-trivial in $H_{2}\left(D_{a}, \mathbb{Z}\right)$ and trivial in $H_{2}(\mathcal{M}, \mathbb{Z})$. Locally the $\mathrm{U}(1)_{Y}$ flux sources an RR tadpole, which is in fact absent globally due to the triviality of the cycle. The finiteness of threshold corrections is tied to the absence of RR tadpoles, but the triviality of $C_{a}$ requires knowledge of the global geometry, leading to the presence of the scale $R M_{s}$.

\subsection{Moduli stabilisation}

So far we essentially considered a local part of the overall Calabi-Yau geometry where the GUT physics is localised. As has been pointed out in [13], supersymmetry breaking on a hidden D-brane and mediation via gauge interaction to the visible GUT brane might also 
partly allow a completely local treatment. This presumes of course that all possible Planck scale suppressed terms are sub-leading.

In this paper, we do not postpone these global issues but instead continue the quite successful investigation of moduli stabilisation in the framework of Type IIB orientifolds. More concretely, we consider a set-up where the bulk moduli orthogonal to $D_{a}$ are stabilised by the LARGE volume scenario and compute the induced soft terms on the $\mathrm{SU}(5)$ brane, respectively the del Pezzo singularity constructions.

\subsubsection{Fixing the non-standard model/GUT cycles}

For self-consistency let us review briefly the main ingredients for the KKLT respectively LARGE volume scenario.

At order $\mathcal{V}^{-2}$ in the large volume expansion, the complex structure moduli and the dilaton are stabilised by a non-trivial $G_{3}$-flux giving rise to a tree-level superpotential of the form [26]

$$
W_{\text {flux }}=\int_{\mathcal{M}} G_{3} \wedge \Omega_{3}
$$

The resulting scalar potential is of the no-scale type, with the Kähler moduli still flat directions.

In the LARGE volume scenario the no-scale structure is broken by a combination of $\alpha^{\prime}$-corrections to the Kähler potential and a D3-instanton correction to the superpotential. Concretely, the Kähler potential including $\alpha^{\prime}$-corrections [27] reads

$$
\mathcal{K}=-2 \ln \left(\mathcal{V}+\frac{\hat{\xi}}{2}\right)-\ln (S+\bar{S})+\mathcal{K}_{\mathrm{CS}},
$$

where $\hat{\xi}=\xi / g_{s}^{3 / 2}$ and $g_{s}$ is the string coupling. The resulting inverse Kähler metric for the Kähler moduli $T_{a}$ and the axio-dilaton $S$ reads

$$
\begin{aligned}
\mathcal{K}^{a \bar{b}} & =-2\left(\mathcal{V}+\frac{\hat{\xi}}{2}\right)\left(\frac{\partial^{2} \mathcal{V}}{\partial \tau_{a} \partial \tau_{b}}\right)^{-1}+\tau_{a} \tau_{b} \frac{4 \mathcal{V}-\hat{\xi}}{\mathcal{V}-\hat{\xi}} \\
\mathcal{K}^{a \bar{S}} & =-\frac{3}{2}(S+\bar{S}) \frac{\hat{\xi}}{\mathcal{V}-\hat{\xi}} \tau_{a}, \\
\mathcal{K}^{S \bar{S}} & =\frac{(S+\bar{S})^{2}}{4} \frac{4 \mathcal{V}-\hat{\xi}}{\mathcal{V}-\hat{\xi}}
\end{aligned}
$$

For a D3-instanton to generate a contribution to the superpotential it has to have the right number of zero modes. In fact an $O(1)$ instanton wrapping a rigid four-cycle, which does not intersect any four-cycle carrying D7-branes, has the right number of zero modes to give a contribution

$$
W=W_{0}+A e^{-a T_{s}}
$$

to the superpotential. Here $W_{0}$ is the value of the GVW superpotential in the minimum and we implicitly assumed that one can first integrate out the complex structure moduli and the axio-dilaton multiplet. This is justified by observing that the instanton induced 
scalar potential is at order $\mathcal{O}\left(\mathcal{V}^{-3}\right)$ in the LARGE volume expansion and that the Kähler metric for the complex structure and Kähler moduli has a factorised form (for discussions of integrating out moduli in supergravity see [28-31]).

Working in the large cycle regime, in the simplest case, one chooses the volume $\mathcal{V}$ of the internal space to be of swiss-cheese form with three Kähler moduli

$$
\mathcal{V}=\left(\eta_{b} \tau_{b}\right)^{3 / 2}-\left(\eta_{s} \tau_{s}\right)^{3 / 2}-\left(\eta_{a} \tau_{a}\right)^{3 / 2}
$$

Here $\tau_{b}$ determines the size of the Calabi-Yau and the small four-cycle of size $\tau_{s}$ is wrapped by the D3-brane instanton. The resulting F-term potential at order $\mathcal{O}\left(\mathcal{V}^{-3}\right)$ reads

$$
\begin{aligned}
V_{F} & =e^{\mathcal{K}}\left(\mathcal{K}^{a \bar{b}} D_{a} W D_{\bar{b}} \bar{W}-3|W|^{2}\right) \\
& =\lambda \frac{(a A)^{2} \sqrt{\tau_{s}} e^{-2 a \tau_{s}}}{\mathcal{V}}-\mu \frac{a\left|A W_{0}\right| \tau_{s} e^{-a \tau_{s}}}{\mathcal{V}^{2}}+\nu \frac{\xi\left|W_{0}\right|^{2}}{g_{s}^{1 / 2} \mathcal{V}^{3}}+\ldots,
\end{aligned}
$$

with coefficients $\lambda=\frac{g_{s}}{2} \frac{8}{3 \eta_{s}^{3 / 2}}, \mu=2 g_{s}, \nu=\frac{3}{8}$, featuring the LARGE volume AdS minimum at $\mathcal{V} \sim e^{a \tau_{s}}$. More details of this minimum are collected in appendix A, which allows one to compute the value of the scalar potential (2.13) in this minimum to be

$$
V_{0}=-\frac{3}{16 a \tau_{s}} \frac{\xi}{g_{s}^{3 / 2}} \frac{W_{0}^{2}}{\mathcal{V}^{3}} M_{P}^{2} .
$$

Clearly it is negative, but due to a cancellation of the leading order terms, it contains an extra suppression by $\left(a \tau_{s}\right) \simeq \log (\mathcal{V})$. For a realistic model this negative vacuum energy has to be uplifted to $V_{0} \simeq 0$. For the computation of the gravity and anomaly mediated soft terms in section 3 , we will start by neglecting the effects of uplifting, but will then consider the contributions of the uplifting sector.

\subsubsection{Fixing the standard model/GUT cycle}

Coming back to the GUT brane, following the zero mode arguments in [7], we assume that the GUT branes are wrapping a four-cycle of size $\tau_{a}$ which is "orthogonal" to the instanton cycle. As mentioned, in Type IIB orientifolds we allow for an additional gauge flux $\mathcal{F}_{a}$ in the diagonal $\mathrm{U}(1)_{a} \subset \mathrm{U}(5)$ perturbative Chan-Paton gauge group. Vanishing of the "Fayet-Iliopoulos" $\mathrm{U}(1)_{a}$ D-term constraint (at order $\mathcal{V}^{-2}$ )

$$
\int_{D_{a}} J \wedge \mathcal{F}_{a}=0
$$

implies that $\tau_{a} \rightarrow 0$ so that one is driven to the quiver locus where $\alpha^{\prime}$-corrections cannot be ignored. In the EFT the condition (2.15) is essentially that the field dependent FI-term vanishes $\mathcal{K}_{T_{a}}=0$. Using the Kähler potential in both the geometric and quiver regimes, this condition show explicitly a dynamical preference for a collapsed cycle $\tau_{a} \rightarrow 0$. The F-term of the field $T_{a}$ is of the form

$$
F_{a}=e^{\mathcal{K} / 2}\left(W_{T_{a}}+W K_{T_{a}}\right) .
$$


Since the superpotential $W$ does not depend on the modulus $T_{a}$ and the D-term condition implies $\mathcal{K}_{T_{a}}=0$, we can see that this field does not break supersymmetry, i. e. $F_{a}=$ 0 . Notice that this conclusion will not be modified by including perturbative and nonperturbative corrections to the Kähler potential since these corrections will equally modify the D- and F-terms. Since $\tau_{a}=0$ one finds that also $F^{a}=0$, which is a very important conclusion, as it indicates that the standard model is somehow sequestered from the sources of supersymmetry breaking.

A loophole to this argument is that it implicitly assumes that the standard model fields, charged under the corresponding U(1), will not get a VEV. Otherwise they would contribute to the D-terms and cancel the contribution from the FI-term. Even though this is desirable phenomenologically to avoid a large scale breaking of the standard model symmetries, such as colour, it should be the outcome of a calculation. We illustrate in the appendix in a toy model that this is actually the case as long as the soft scalar masses are not tachyonic.

A direct consequence is that the soft terms on the GUT brane can only be generated at "sub-leading" order by $F^{b}, F^{s}$ and $F^{S}$, i. e. by moduli which are sort of sequestered from the GUT brane.

\subsection{Including matter fields}

So far we have concentrated only on the EFT for moduli fields and their stabilisation. In order to study soft-supersymmetry breaking we need to properly introduce the matter field dependence in the EFTs in both the geometric and singular cycle regimes. The important term to be included is the matter fields' Kähler potential $\tilde{K}=Z_{\alpha \beta} \varphi_{\alpha} \varphi_{\beta}^{*}+\cdots$ with $Z_{\alpha \beta}$ a function of the moduli fields.

At this state, only the dependence on $\tau_{b}$ and $\tau_{s}$ is relevant, as all the other fields do not break supersymmetry (to leading order). $Z$ should only depend on $\tau_{b}, S$ and the Kähler modulus of the GUT brane $\tau_{a}$, so $Z=Z\left(\tau_{b}, \tau_{a}, S\right)$. The leading order expression for $Z$ was determined in [32] with $Z \sim 1 / \mathcal{V}^{2 / 3}$ (see also [33]) which applies to both chiral matter at magnetised D7-branes and to the better understood fractional D3-branes at singularities. Since the $\alpha^{\prime}$-corrections to the Kähler potential are crucial to determine the large volume vacuum, consistency requires that these corrections should also be included in the matter field Kähler potential. Unfortunately these corrections are not known at present. However, as in the tree-level case, we are mostly interested on their overall volume dependence.

Let us parametrise the $\alpha^{\prime}$-corrections by a so far unknown function $f$ :

$$
Z_{\alpha}=\frac{k_{\alpha}}{\tau_{b}}\left(1+f\left(\frac{\operatorname{Re}(S)}{\tau_{b}}\right)\right) .
$$

The dependence of $f$ on the variables can only be in the indicated way in order to have the right power in $g_{s}$. Now consider the next-to-leading order correction in $\alpha^{\prime}$ to the tree-level result, which, we claim, must be of the form:

$$
Z_{\alpha}=\frac{k_{\alpha}}{\tau_{b}}\left(1-\delta\left(\frac{\operatorname{Re}(S)}{\tau_{b}}\right)^{\frac{n}{2}}+\cdots\right),
$$


with $n=1,2, \ldots$ denoting the $\left(\alpha^{\prime}\right)^{n}$ order of this term. The question now is at which order in $\left(\alpha^{\prime}\right)^{n}$ the first correction appears. Since we are only interested in the correction which does not include $\tau_{a}$, we can use a scaling argument like in [32]. Assuming that the physical Yukawa couplings do not depend on the overall volume of the space and taking into account the Kähler potential (2.9), the leading order correction to the Kähler metrics were shown to scale as $\frac{k_{\alpha}}{\tau_{b}}$. Then it is expected that also at next-to-leading order the scalings must match, which means that also the Kähler metrics are corrected at order $\left(\alpha^{\prime}\right)^{3}$. This argument shows that $n=3$ is the smallest expected correction in (2.18) and then $Z_{\alpha}=\frac{k_{\alpha}}{\tau_{b}}\left(1-\delta\left(\frac{\operatorname{Re}(S)}{\tau_{b}}\right)^{3 / 2}\right)$.

\subsection{Summary of EFTs}

We can finally summarise the expressions for the EFTs we are using for the two relevant regimes:

1. In the geometric regime the EFT is determined by:

$$
\begin{aligned}
\mathcal{K} & =-2 \ln \left(\mathcal{V}+\frac{\hat{\xi}}{2}\right)-\ln (S+\bar{S})+\mathcal{K}_{\mathrm{CS}}+Z \varphi \varphi^{*}+\cdots, \\
W & =W_{0}+A e^{-a T_{s}}+W_{\text {matter }} \\
f_{i} & =T_{a}-\frac{1}{2} \kappa_{i} S
\end{aligned}
$$

where $\mathcal{V}=\left(\eta_{b} \tau_{b}\right)^{3 / 2}-\left(\eta_{s} \tau_{s}\right)^{3 / 2}-\left(\eta_{a} \tau_{a}\right)^{3 / 2}$ and $Z=k\left(1-\delta(\operatorname{Re}(S))^{3 / 2} / \mathcal{V}\right) / \mathcal{V}^{2 / 3}$.

2. In the singular cycle (blow-up) regime there is a slight change in the standard model cycle dependence of $\mathcal{K}$ :

$$
\begin{aligned}
\mathcal{K} & =-2 \ln \left(\mathcal{V}+\frac{\hat{\xi}}{2}\right)+\alpha \frac{\tau_{a}^{2}}{\mathcal{V}}-\ln (S+\bar{S})+\mathcal{K}_{\mathrm{CS}}+Z \varphi \varphi^{*}+\cdots, \\
W & =W_{0}+A e^{-a T_{s}}+W_{\text {matter }} \\
f & =\delta_{i} S+s_{i k} T_{k},
\end{aligned}
$$

with now $\mathcal{V}=\left(\eta_{b} \tau_{b}\right)^{3 / 2}-\left(\eta_{s} \tau_{s}\right)^{3 / 2}$ and $Z=\left(\beta-\delta / \mathcal{V}+\gamma \tau_{a}^{m}\right) / \mathcal{V}^{2 / 3}$ with $m>0$

Since in both cases the standard model/GUT cycle does not break supersymmetry, the structure of soft breaking terms will be essentially the same.

\section{Gravity mediated soft terms}

As we have seen, the LARGE volume minimum of the scalar potential breaks supersymmetry, so that this breaking induces soft supersymmetry breaking terms on the GUT brane. There are two sources which are relevant here. First, there are of course the gravity mediated soft terms. However, since the GUT brane is sequestered from the non-supersymmetric bulk one might expect that anomaly mediation is the leading order contribution. In this 
section we compute the gravity mediated soft terms, i. e. the gaugino- and sfermion-masses as well as the $\mu_{-}, \mathrm{A}$ - and B-terms. Moreover, we compute the anomaly mediated gaugino masses. Let us emphasise again that the scenario differs from the usual intermediate scale LARGE volume scenario in that the string scale is much higher (we assume $M_{s} \sim 10^{15} \mathrm{GeV}$ for consistency with unification at $M_{X} \sim 10^{16} \mathrm{GeV}$ ), and that the GUT or MSSM branes are wrapping a four-cycle completely sequestered from the four-cycles supporting D3-brane instantons.

First we express the string scale $M_{s}=\left(\alpha^{\prime}\right)^{-1 / 2}$ in terms of the Planck scale and the volume $\mathcal{V}$ of internal Calabi-Yau (in Einstein frame and in units of $\ell_{s}=2 \pi \sqrt{\alpha^{\prime}}$ )

$$
M_{s}=\frac{\sqrt{\pi} g_{s}^{1 / 4}}{\sqrt{\mathcal{V}}} M_{P} .
$$

Thus we obtain $M_{s} \simeq 10^{15} \mathrm{GeV}$ and $M_{X} \simeq 1.2 \cdot 10^{16} \mathrm{GeV}$ for $\mathcal{V}=\mathcal{O}\left(10^{6}-10^{7}\right)$, a value large enough to trust the $\mathcal{V}^{-1}$ expansion. Moreover, we immediately realise that the LARGE volume expansion parameter is directly related to the local GUT expansion parameter, i.e. $\mathcal{V}^{-1 / 2} \simeq M_{s} / M_{P}$. For computing the gravitino mass we simply utilise the general formula $M_{3 / 2}=e^{\frac{\mathcal{K}}{2}} W$ leading in our case to

$$
M_{3 / 2}=\frac{g_{s}^{1 / 2}\left|W_{0}\right|}{\sqrt{2} \mathcal{V}} M_{P} .
$$

\subsection{Structure of soft terms}

We are now in a position to compute each of the gravity mediated soft supersymmetry breaking terms in this class of scenarios.

\subsubsection{Gaugino masses}

For gravity mediated supersymmetry breaking, the gaugino masses are calculated as

$$
M_{\widetilde{G}}=\frac{1}{2 \operatorname{Re}\left(f_{i}\right)} F^{I} \partial_{I} f_{i}
$$

for $i=3,2,1$, where for the gauge kinetic functions we use (2.2) with $\tau_{a} \simeq 0$ due to the D-term constraint.

Since the GUT brane is sequestered from the bulk we have $F^{a}=0$ and the only contribution can come from the dilaton $\mathrm{F}$-term $F^{S}=e^{\mathcal{K} / 2} \mathcal{K}^{S \bar{J}} \bar{F}_{\bar{J}}$. We assume that the F-term condition for the axio-dilaton $F_{S}=0$ is fulfilled at leading order. At next-toleading order, there are then only sub-leading contributions from $F_{S}$ as well as terms from $F_{b}$ adding up to $F^{S}=\frac{3}{2 \sqrt{2}} \gamma \frac{\xi}{g_{s}^{2}} \frac{W_{0}}{\mathcal{V}^{2}}$ where $\gamma$ is an $\mathcal{O}(1)$ factor (see in the appendix for a more detailed derivation). Thus, the gravity mediation induced term for the gaugino masses reads:

$$
M_{\widetilde{G}}=\frac{3}{4 \sqrt{2}} \gamma \frac{\xi}{g_{s}} \frac{\left|W_{0}\right| M_{P}}{\mathcal{V}^{2}}=\frac{3}{4} \gamma \frac{\xi}{g_{s}^{3 / 2}} \frac{M_{3 / 2}}{\mathcal{V}},
$$

independent of the MSSM gauge group factor, as the factor $\kappa_{i}$ in (2.2) cancels. Here we have assumed that the D-term fixes the size of the GUT four-cycle at small volume in string units, so that the leading contribution to $\operatorname{Re}\left(f_{i}\right) \simeq 25$ comes from the gauge flux induced correction $\simeq \kappa_{i} \operatorname{Re}(S)$. 


\subsubsection{Squark/slepton masses}

The scalar masses obtained for gravity mediation of supersymmetry breaking read

$$
M_{\widetilde{Q}}^{2}=M_{3 / 2}^{2}+V_{0}-F^{I} F^{\bar{J}} \partial_{I} \partial_{\bar{J}} \ln Z_{\alpha},
$$

where the potential in the minimum $V_{0}$ is assumed to be already uplifted so that $V_{0} \simeq 0$.

Computing now the soft-sfermion masses, let us first discuss the tree-level term in $Z_{\alpha}$. In this case (3.5) reduces to

$$
M_{\widetilde{Q}}^{2}=M_{3 / 2}^{2}-\frac{\left(F^{b}\right)^{2}}{4 \tau_{b}^{2}},
$$

where we have neglected the vacuum energy in the minimum. Again, there is a cancellation of the gravitino mass squared with the leading term in $\left(F^{b}\right)^{2}$. The term quadratic in $F^{S}$ is sub-leading as being of order $\mathcal{V}^{-4}$. In the appendix A we compute the next-to-leading order term in $F^{b}$, which reads

$$
\left(F^{b}\right)^{2} \approx 4 \tau_{b}^{2}\left[M_{3 / 2}^{2}+\frac{3}{8 a \tau_{s}} \frac{\xi}{g_{s}^{3 / 2}}\left(1+\frac{3}{2 a \tau_{s}}\right) \frac{M_{3 / 2}^{2}}{\mathcal{V}}\right]
$$

Therefore, one gets for the soft sfermion masses squared

$$
\begin{aligned}
M_{\widetilde{Q}}^{2} & =-\frac{3}{16 a \tau_{s}} \frac{\xi}{g_{s}^{1 / 2}}\left(1+\frac{3}{2 a \tau_{s}}\right) \frac{\left|W_{0}\right|^{2} M_{P}^{2}}{\mathcal{V}^{3}} \\
& =-\frac{3}{8 a \tau_{s}} \frac{\xi}{g_{s}^{3 / 2}} \frac{M_{3 / 2}^{2}}{\mathcal{V}}
\end{aligned}
$$

which at this stage come out tachyonic.

Next we need to discuss the higher $\alpha^{\prime}$-corrections in (2.18). The term with the highest power in $1 / \mathcal{V}$ is the one with $\left(F^{b}\right)^{2} \partial_{b} \partial_{b} \log \cdots$. It is straightforward, that for $\tau_{a} / \tau_{b} \ll 1$ this simplifies to

$$
F^{m} F^{n} \partial_{m} \partial_{n} \log \left(1-\delta\left(\frac{\operatorname{Re} S}{\tau_{b}}\right)^{\frac{n}{2}}+\cdots\right) \simeq F^{b} F^{b} \frac{\delta n(n+2)(\operatorname{Re} S)^{\frac{n}{2}}}{4 \tau_{b}^{\frac{n}{2}+2}} \sim \frac{\delta}{g_{s}^{\frac{n-2}{2}}} \frac{\left|W_{0}\right|^{2} M_{P}^{2}}{\mathcal{V}^{\left(2+\frac{n}{3}\right)}}
$$

Therefore, if there would be corrections of order $n=1,2$, they would dominate over the corrections in (3.8). It is precisely the third order corrections in $\alpha^{\prime}$ which contribute to the sfermion masses at the same order in $1 / \mathcal{V}$. Including also the other moduli fields in (3.9), the overall value of the squared scalar masses will then be proportional to $\delta-\xi / 3$ :

$$
M_{\widetilde{Q}}^{2}=M_{3 / 2}^{2}\left(-\frac{1}{4 a \tau_{s}} \frac{\xi}{g_{s}^{3 / 2} \mathcal{V}}+\frac{15(\delta-\xi / 3)}{4 g_{s}^{3 / 2} \mathcal{V}}\right)
$$

Therefore, depending on the relative size of these two contributions one can get tachyonic or non-tachyonic sfermion masses. Moreover, it also shows that for $\delta=\xi / 3$ there are further cancellations taking place at this order. This is precisely the value one expects from the above mentioned scaling argument of the physical Yukawa couplings. Later we 
will give an argument under which quite general assumptions such cancellations should occur. One of the assumptions will be that really the uplifting sector is correctly taken into account, which leads to a further dependence of the Kähler metric on a supersymmetry breaking field. Note that indeed the soft sfermion masses (3.8) are of the same order as the AdS vacuum energy (2.14), indicating that in these computations the uplift sector cannot be neglected.

\subsection{3 $\hat{\mu} / \hat{\mu} B$-terms}

The formula for the $\hat{\mu}$-term is

$$
\hat{\mu}=\left(e^{\mathcal{K} / 2} \mu+M_{3 / 2} Z-\bar{F}^{\bar{I}} \partial_{\bar{I}} Z\right)\left(Z_{H_{1}} Z_{H_{2}}\right)^{-1 / 2},
$$

where $\mu$ denotes the supersymmetric $\mu$-parameter, which we keep for completeness, although it can be argued to vanish under very general assumptions [32]. We assume again the Kähler metric (2.18) for the Higgs fields as well as for $Z$. Here again, a cancellation of the second and the third term occurs. Note, if the $\mu$ parameter is not equal to zero, it dominates over the sub-leading terms stemming from $F^{b}$. Dropping the factors of order one, we are left with:

$$
\hat{\mu} \approx \frac{\sqrt{g_{s}}}{\sqrt{2}} \frac{\tau_{b}}{\mathcal{V}} \mu-\frac{M_{\widetilde{G}}}{4 a \tau_{s}} .
$$

The expression for $B \hat{\mu}$ is more complicated:

$$
\begin{aligned}
B \hat{\mu}=\left(Z_{H_{1}} Z_{H_{2}}\right)^{-1 / 2} & \left(e^{\mathcal{K} / 2} \mu\left(F^{I} \partial_{I} \mathcal{K}+F^{I} \partial_{I} \log \mu-F^{I} \partial_{I} \log \left(Z_{H_{1}} Z_{H_{2}}\right)-M_{3 / 2}\right)\right. \\
& +\left(2 M_{3 / 2}^{2}+V_{0}\right) Z-M_{3 / 2} \bar{F}^{\bar{I}} \partial_{\bar{I}} Z+M_{3 / 2} F^{I}\left(\partial_{I} Z-Z \partial_{I} \log \left(Z_{H_{1}} Z_{H_{2}}\right)\right) \\
& \left.-F^{\bar{I}} F^{J}\left(\partial_{\bar{I}} \partial_{J} Z-\left(\partial_{\bar{I}} Z\right) \partial_{J} \log \left(Z_{H_{1}} Z_{H_{2}}\right)\right)\right) .
\end{aligned}
$$

However, due to the simple Kähler metric and assuming that $\mu$ is just an input parameter without any moduli dependence, after a long but straightforward calculation, the result is rather simple:

$$
B \hat{\mu}=-\left(\frac{\sqrt{g_{s}}}{\sqrt{2}} \frac{\tau_{b}}{\mathcal{V}} \mu+\frac{M_{3 / 2}}{2 a \tau_{s}}\right) M_{\widetilde{G}},
$$

where we have dropped again the order one constants $k_{H_{i}}$ and $z$.

\subsubsection{A-terms}

The A-terms are given by:

$$
A_{\alpha \beta \gamma}=F^{I}\left(\partial_{I} \mathcal{K}\right)+F^{I} \partial_{I} \log Y_{\alpha \beta \gamma}-F^{I} \partial_{I} \log Z_{\alpha} Z_{\beta} Z_{\gamma} .
$$

The Peccei-Quinn shift-symmetry forbids a dependence of the holomorphic superpotential on the axio-dilaton or Kähler moduli, thus the Yukawa couplings $Y_{\alpha \beta \gamma}$ can only depend on the complex structure moduli and they drop out.

There is a cancellation of $F^{b}$ in the remaining two sums and we are left with

$$
A_{\alpha \beta \gamma}=F^{s}\left(\partial_{s} \mathcal{K}\right)+F^{S}\left(\partial_{S} \mathcal{K}\right) .
$$


As listed in the appendix, $F^{s}\left(\partial_{s} \mathcal{K}\right)$ is suppressed with respect to $F^{S}\left(\partial_{S} \mathcal{K}\right)$ by a factor of $1 / a \tau_{s}$. As we are interested only in orders of magnitude, we keep only the latter term and get as result:

$$
A_{\alpha \beta \gamma} \approx F^{S}\left(\partial_{S} \mathcal{K}\right)=-\frac{3}{4 \sqrt{2}} \frac{\xi}{g_{s}} \frac{\left|W_{0}\right|}{\mathcal{V}^{2}} M_{P}=-M_{\widetilde{G}}
$$

\subsubsection{Anomaly mediated gaugino masses}

Let us also now estimate the anomaly mediated gaugino mass. It is clear that, for such a sequestered observable sector, one would have guessed that not gravity mediation but anomaly mediation induces the leading order soft terms. General formula for all the different soft terms are not available, so that in this section we just compute the anomaly mediated gaugino masses. The expression for them reads [34] (see also [35]): ${ }^{2}$

$$
\begin{aligned}
M_{\widetilde{G}}^{\mathrm{anom}}=-\frac{g^{2}}{16 \pi^{2}}\left[\left(3 T_{G}-T_{R}\right) M_{3 / 2}-\right. & \left(T_{G}-T_{R}\right)\left(\partial_{I} \mathcal{K}\right) F^{I} \\
& \left.-\frac{2 T_{R}}{d_{R}} F^{I} \partial_{I} \log \operatorname{det} Z_{\alpha \beta}\right],
\end{aligned}
$$

where $T_{G}$ is the Dynkin index of the adjoint representation, normalised to $N$ for $\mathrm{SU}(N)$, and $T_{R}$ is the Dynkin index associated with the representation $R$ of dimension $d_{R}$, normalised to $1 / 2$ for the $\mathrm{SU}(N)$ fundamental.

A careful calculation of $F^{b}$, worked out in the appendix, reveals that it is proportional to the gravitino mass at leading order: $F^{b} \approx-2 \tau_{b} M_{3 / 2}-\frac{\tau_{b}}{2 a \tau_{s}} M_{\widetilde{G}}$. This leads to a precise cancellation of $M_{3 / 2}$ in (3.18). The final expression for the anomaly mediated gaugino mass for a $\mathrm{SU}(N)$ gauge group is thus

$$
M_{\widetilde{G}}^{\text {anom }}=-\frac{g^{2}}{16 \pi^{2}}\left[\left(N-\frac{1}{2}\right)-\frac{1}{4 a \tau_{s}}\left(3 N-\frac{1}{2}\right)\right] M_{\widetilde{G}} .
$$

Surprisingly, though the gravity mediated contribution to the gaugino mass is suppressed with respect to the gravitino mass by a factor of $\left(M_{X} / M_{P}\right)^{2}$, anomaly mediation is not the dominating source for the gaugino mass. It is suppressed by the usual one-loop factor with respect to the gravity mediated contribution. One expects a similar suppressed behaviour for the other soft terms, so that anomaly mediation is even sub-leading to gravity mediation.

\subsection{Summary of gravity mediated soft masses}

In the above computation of soft terms we have seen that the leading terms cancel and that we need to include higher order corrections in $\mathcal{V}^{-1}$. Since this scale is directly correlated with $\zeta=M_{s} / M_{P}$, we can express these gravity mediated soft terms in terms of the scales $M_{3 / 2}$ and $\zeta=M_{s} / M_{P}$. The results are listed in table 1 , where we have set the supersymmetric $\mu$ parameter to zero and estimated

$$
\sqrt{\frac{\pi}{3 \xi}} \simeq \sqrt{\frac{400}{\chi(\mathcal{M})}} \simeq 1, \quad \text { and } \quad g_{s} \simeq 1
$$




\begin{tabular}{|c|c|}
\hline soft-term & scale \\
\hline$M_{\widetilde{G}}$ & $\frac{1}{4} M_{3 / 2} \zeta^{2}$ \\
\hline$M_{\widetilde{Q}}^{2}$ & $\frac{1}{16 \log \zeta} M_{3 / 2}^{2} \zeta^{2}$ \\
\hline$\hat{\mu}$-term & $\frac{1}{8 \log \zeta} M_{\widetilde{G}}$ \\
\hline$B \hat{\mu}$-term & $\frac{1}{4 \log \zeta} M_{3 / 2} M_{\widetilde{G}}$ \\
\hline$A$-term & $-M_{\widetilde{G}}$ \\
\hline
\end{tabular}

Table 1. Classical gravity mediated soft terms for a naïve computation of soft terms. Here the expansion parameter is $\zeta=M_{s} / M_{P}$. We have assumed the supersymmetric $\mu$-term to vanish [32].

All soft terms in table 1 are suppressed by $\left(M_{s} / M_{P}\right)^{2}$ relative to the naïve expectation $M_{3 / 2}^{n}$ with $n=1,2$ depending on the mass-dimension. This explicitly demonstrates that gravity effects from the bulk are suppressed on the shrinkable GUT cycles, which is the main assumption of the local F-theory GUTs.

However, as seen in the text in certain cases there can be more cancellations leading to even higher suppressions. Indeed so far we have neglected the uplift sector, but have seen that the sfermion masses are actually of the same order of magnitude as the uplift so that it should better not be neglected. We now discuss under which well-posed assumptions further cancellations are present.

\subsection{Uplift and cancellations}

In the last section we have computed the gravity induced soft terms on the GUT brane. As we have explained, the computation relies on assumptions about the expansions of the matter metrics at higher orders in $\alpha^{\prime}$. While such corrections must surely be present, it is difficult to know the precise form of these corrections. We have explicitly seen for the sfermion masses that these corrections contribute at the same order in $1 / \mathcal{V}$ as the next-toleading order contributions from $F^{b}$. Indeed, as seen in eq. (3.10) there can potentially be further cancellations at this order. We have also computed the soft terms under the assumption of $V_{0}=0$, but have not taken into account the contribution of the supersymmetry breaking from the uplifting sector to the soft terms. To consider these possibilities, let us argue in this section, how one can arrive at quite general statements by making some well posed assumptions and exploiting the consequences of using the supergravity formalism.

Recall that the physical Yukawas are given by

$$
\hat{Y}_{\alpha \beta \gamma}=e^{\mathcal{K} / 2} \frac{Y_{\alpha \beta \gamma}}{\sqrt{Z_{\alpha} Z_{\beta} Z_{\gamma}}} .
$$

\footnotetext{
${ }^{2}$ We use a different sign convention for the F-terms leading to a different sign in the second and third term in the anomaly mediated mass term than in [34].
} 
The shift-symmetries of the Kähler moduli imply that they do not appear perturbatively in the superpotential Yukawa couplings $Y_{\alpha \beta \gamma}$. Let us make the assumption that the physical Yukawas, being local renormalisable couplings, do not depend on the fields breaking supersymmetry. This includes the volume and also the hidden sector fields that are responsible for uplifting and giving vanishing cosmological constant. We also assume pure F-term uplifting.

Such supersymmetry breaking fields appear in the overall Kähler potential, and the constraints of holomorphy then imply that in order for the physical Yukawas to be independent of such fields,

$$
Z_{\alpha}=e^{\mathcal{K} / 3}
$$

Note that this includes the tree-level behaviour of local matter fields, $Z_{\alpha} \sim \frac{1}{\mathcal{V}^{2 / 3}} \sim \frac{1}{\left(T_{b}+T_{b}\right)}$. In this case it follows that

$$
\begin{aligned}
m_{Q}^{2} & =V_{0}+M_{3 / 2}^{2}-F^{m} \bar{F}^{\bar{n}} \partial_{m} \partial_{\bar{n}} \ln Z_{\alpha} \\
& =V_{0}+M_{3 / 2}^{2}-F^{m} \bar{F}^{\bar{n}} \frac{\mathcal{K}_{m \bar{n}}}{3}=\frac{2}{3} V_{0}=0,
\end{aligned}
$$

for the case of vanishing cosmological constant.

The A-terms also vanish under this assumption. In this case the A-terms can be most intuitively written as

$$
A_{\alpha \beta \gamma} Y_{\alpha \beta \gamma}=F^{I} \partial_{I} \hat{Y}_{\alpha \beta \gamma}
$$

with $\hat{Y}_{\alpha \beta \gamma}$ the physical Yukawa couplings. So it immediately follows that if the physical Yukawa couplings do not depend on the fields breaking supersymmetry, the A-terms all vanish.

The anomaly mediated contribution for gaugino masses gives

$$
\begin{aligned}
M_{\widetilde{G}}^{\text {anom }} & =\frac{b_{a}}{16 \pi^{2}} M_{3 / 2}-\frac{\left(\sum_{r} n_{r} T_{a}(r)-T(G)\right)}{16 \pi^{2}} F^{m} \partial_{m} \mathcal{K}(\Phi, \bar{\Phi})+\sum_{r} \frac{n_{r} T_{a}(r)}{8 \pi^{2}} F^{m} \partial_{m} \ln Z^{r}(\Phi, \bar{\Phi}) \\
& =\frac{b_{a}}{16 \pi^{2}} M_{3 / 2}-\frac{\left(\sum_{r} n_{r} T_{a}(r)-3 T(G)\right)}{16 \pi^{2}} \frac{F^{m} \partial_{m} \mathcal{K}(\Phi, \bar{\Phi})}{3} \\
& =\frac{b_{a}}{16 \pi^{2}}\left(M_{3 / 2}-\frac{1}{3} F^{m} \partial_{m} \mathcal{K}\right)
\end{aligned}
$$

where we have used $Z=e^{\mathcal{K} / 3}$. The size of the anomaly mediated contributions to gaugino masses then depends on the size of $M_{3 / 2}-\frac{1}{3} F^{m} \partial_{m} \mathcal{K}$. The no-scale cancellation for $\tau_{b}$ implies the $\mathcal{O}\left(\mathcal{V}^{-1}\right)$ terms cancel with non-vanishing terms at $\mathcal{O}\left(\mathcal{V}^{-2}\right)$. However (3.25) also includes the hidden uplifting sector, which must have $\mathcal{K}_{\phi \bar{\phi}} F^{\phi} F^{\bar{\phi}} \sim \frac{1}{\mathcal{V}^{3}}$ (in order to uplift the vacuum energy to Minkowski). At this level we therefore cannot rule out that $F^{\phi} \partial_{\phi} \mathcal{K} \sim \mathcal{V}^{-3 / 2}$, giving gaugino masses of order $\frac{g^{2}}{16 \pi^{2}} \frac{1}{\mathcal{V}^{3 / 2}}$.

For the $\mu$-term, we obtain

$$
\hat{\mu}=e^{\mathcal{K} / 6} \mu+\left(M_{3 / 2}-\frac{1}{3} F^{I} \partial_{I} \mathcal{K}\right)
$$


For the B-term, we have (assuming no moduli dependence in $\mu$ )

$$
\begin{aligned}
(B \mu)= & \left(Z_{H_{1}} Z_{H_{2}}\right)^{-1 / 2}\left(e^{\mathcal{K} / 2} \mu\left(F^{I} \partial_{I} \mathcal{K}-F^{I} \partial_{I} \ln \left(Z_{H_{1}} Z_{H_{2}}\right)-M_{3 / 2}\right)\right. \\
& +\left(2 M_{3 / 2}^{2}+V_{0}\right) Z-M_{3 / 2} F^{\bar{I}} \partial_{\bar{I}} Z+M_{3 / 2} F^{I}\left(\partial_{I} Z-Z \partial_{I} \ln \left(Z_{H_{1}} Z_{H_{2}}\right)\right) \\
& \left.-F^{I} F^{\bar{J}}\left(\partial_{\bar{I}} \partial_{J} Z-\left(\partial_{\bar{I}} Z\right) \partial_{J} \ln \left(Z_{H_{1}} Z_{H_{2}}\right)\right)\right) .
\end{aligned}
$$

If we take $Z=Z_{H_{1}}=Z_{H_{2}}=e^{\mathcal{K} / 3}$ then we eventually obtain

$$
B \mu=e^{\mathcal{K} / 6} \mu\left(\frac{1}{3} F^{I} \partial_{I} \mathcal{K}-M_{3 / 2}\right)+\left|\frac{1}{3} F^{I} \partial_{I} \mathcal{K}-M_{3 / 2}\right|^{2} .
$$

This implies that the $\mu$ - and B-terms involve the same expression as appeared in the anomaly mediated expression (3.25) and that the $\mu$ - and $B \mu$-term are of the same order as required for successful electroweak symmetry breaking.

As we do not currently know the form of $\alpha^{\prime}$-corrections to the matter metrics, we do not know whether the form $Z=e^{\mathcal{K} / 3}$ is correct. However it is a natural choice in the sense that it simply says that the physical Yukawa couplings, being local, do not depend on the value of bulk fields. In the context of the $\zeta(3) \chi(\mathcal{M}) \alpha^{\prime 3}$-correction that entered the moduli stabilisation, this is equivalent to the statement that physical Yukawa couplings do not alter if you perform a conifold transition in the bulk (which alters the Calabi-Yau Euler number).

The advantage of phrasing the computation in this way is that we can say that moduli generate soft scalar masses to the extent to which the physical Yukawa couplings depend on the moduli. While not straightforward, it is in principle easier to compute the dependence of physical Yukawa couplings on the moduli. String CFT computations give the directly physical couplings and therefore one could analyse for certain local models (for example for a stack of D3-branes at an orbifold singularity in a compact space) whether the physical couplings do depend on the volume through a direct vertex operator string computation.

We can also use (3.23) to compute the minimal value of the soft scalar masses. The complete cancellation in (3.23) arose from the assumption that the physical Yukawa couplings has no dependence on all fields with non-zero F-terms. However we know this statement is not true. The dilaton has an irreducible F-term of $\mathcal{O}\left(\mathcal{V}^{-2}\right)$ and enters the physical Yukawas. This provides a minimal value for the scale of the physical Yukawa couplings.

\section{Consequences for supersymmetry breaking}

In this previous section we have seen that both gravity and anomaly mediated contributions to soft terms occur at levels far lower than naïve expectation. This gives novel phenomenological consequences for various aspects of supersymmetry breaking, which we now discuss. 


\subsection{Gauge mediated scenarios}

In local F-theory models an interesting proposal was made assuming a model of gauge mediation to dominate supersymmetry breaking in the observable sector. This is very interesting since it incorporates the positive properties of gauge mediation, such as positive squared scalar masses and flavour universality and yet address its problems, such as the $\mu / B \mu$ problem. This proposal though requires the following implicit assumptions:

1. The mechanism responsible for moduli stabilisation, which was not considered, fixes moduli at a high mass and decouples from supersymmetry breaking.

2. Introduce a new matter sector that breaks supersymmetry dynamically and a set of messengers that communicate this breaking to the standard model fields.

3. An anomalous $\mathrm{U}(1)$ was proposed to communicate both sectors and address the $\mu / B \mu$ problem of gauge mediation. The anomalous $\mathrm{U}(1)$ is naturally as heavy as the string scale but has low-energy implications after being integrated out.

These conditions look at first sight too strong and unnatural. Achieving moduli stabilisation without supersymmetry breaking and small cosmological constant is a very strong assumption not realised in any of the moduli stabilisation scenarios so far. It is known that a supersymmetric vacuum in supergravity, such as in KKLT before the uplifting, is naturally anti de Sitter since in that case the vacuum energy is $V_{0}=-3 M_{3 / 2}^{2} M_{P}^{2}$, which is very large unless the superpotential is tuned in such a way that it almost vanishes at the supersymmetric minimum. Also a positive cosmological constant has to be induced after supersymmetry breaking. If the local supersymmetry breaking is responsible for this lifting then its effect should not have been neglected for moduli stabilisation in the first place. Finally, it is not consistent to consider the low-energy effects of a very heavy anomalous $\mathrm{U}(1)$ without also including the effects of the moduli fields which are generically much lighter than the string or compactification scale. In particular the Fayet-Iliopoulos term of the anomalous $\mathrm{U}(1)$ is a function of the moduli.

Nevertheless, our explicit results here show that a scenario similar to this may not be impossible to realise. The main point is that although moduli are stabilised at a nonsupersymmetric point, the breaking of supersymmetry is suppressed by inverse powers of the volume or equivalently by powers of $M_{s} / M_{P}$. This makes the first point above approximately correct. The second point still has to be assumed as in all models of gauge mediation and requires an explicit realisation. Here the relevant observation is to compare the strength of gauge mediation $F_{X} / x$ to the strength of gravity mediation which is usually taken to be $M_{3 / 2}$. However as we have seen the proper comparison is between $F_{X} / x$ with the size of the gravity mediation soft breaking terms which are much smaller than the gravitino mass. Regarding the third point an explicit analysis should be performed in which both the anomalous $\mathrm{U}(1)$ and the moduli are taken into account in the process of moduli stabilisation and supersymmetry breaking.

Very similar to the recently discussed local F-theory models, we may expand our model assuming that there exists a source for gauge mediation, which is parametrised by the 
vacuum expectation values of a scalar field $\langle X\rangle=x+\theta^{2} F_{X}$. This supersymmetry breaking happens in a sector hidden from the GUT brane and is being mediated by messenger fields, which are charged under the GUT gauge group. In order not to spoil gauge coupling unification, this is generically assumed to be a vector-like pair in the $5+\overline{5}$ representation of $\mathrm{SU}(5)$. For our purposes, in this paper we won't present a viable dynamical stringy realisation of this supersymmetry breaking, but just assume that there exists an extra sector, which stabilises the new moduli such that just the field $F_{X}$ develops a non-zero VEV without spoiling the LARGE volume minimum for the bulk moduli. This is clearly a strong assumption, as a dynamical realisation of gauge mediation is known to be challenging [3638]. We will comment more on this towards the end of this section.

The gauge mediated gaugino and sfermion masses are of order

$$
M_{\widetilde{Q}}^{\text {gauge }} \sim M_{\widetilde{G}}^{\text {gauge }}=\frac{\alpha_{X}}{4 \pi} \frac{F_{X}}{x},
$$

where the $\alpha_{X} / 4 \pi$ prefactor is due to the fact that these masses are induced via a one-loop effect for the gauginos and via a two-loop diagram for the sfermions. Note that these formulae used a canonical normalised superfield $X$.

Now, we would like these gauge mediated soft masses to dominate the gravity mediated ones. In particular, we want the gauge mediated sfermion masses to dominate over the gravity mediated ones. To get a first impression of the numerology we get, we also impose the strong constraint that the supersymmetry breaking $F_{X}$ already uplifts the negative vacuum energy (2.14) of the LARGE volume minimum. We therefore require

$$
\frac{F_{X}^{2}}{M_{P}^{2}} \simeq \frac{M_{3 / 2}^{2}}{16 \log \left(\frac{M_{P}}{M_{s}}\right)} \frac{M_{s}^{2}}{M_{P}^{2}},
$$

where $M_{s}$ is the string scale, leading to the relation

$$
F_{X} \simeq \frac{1}{4 \sqrt{\log \left(\frac{M_{P}}{M_{s}}\right)}} M_{3 / 2} M_{s}
$$

Requiring now that $M_{\widetilde{Q}}^{\text {gauge }}>\left|M_{\widetilde{Q}}^{\text {grav }}\right|$ leads to the moderate bound

$$
x<\frac{\alpha_{X}}{4 \pi} M_{P} \simeq 10^{16} \mathrm{GeV},
$$

where we used the relation (3.8). If there is a further suppression, i. e. $M_{\widetilde{Q}} \simeq M_{3 / 2} / \mathcal{V}$, then this bound becomes even more relaxed. For solving the hierarchy problem, one also needs $F_{X} / x \simeq 10^{5} \mathrm{GeV}$. Once one has specified the favourite values for $x$ and $F_{X}$, one can use (4.3) to determine the value of the gravitino mass, which we would like to stress will be gravity-dominated. Let us discuss two examples.

- In the local F-theory models, it was argued that the best values are

$$
x \simeq 10^{12} \mathrm{GeV}, \quad F_{X} \simeq 10^{17} \mathrm{GeV}^{2}
$$


which lead to $M_{3 / 2} \simeq 1 \mathrm{TeV}$, which needs a certain amount of tuning of $W_{0}$. However, the light modulus $\tau_{b}$ has a mass of the order

$$
M_{\tau_{b}} \simeq M_{3 / 2} \frac{M_{s}}{M_{P}},
$$

which in this case gives $M_{\tau_{b}} \simeq 1 \mathrm{GeV}$. For such a light modulus, we expect to face the cosmological modulus problem (CMP).

- Let us now require that the light modulus avoids the CMP by having a mass $M_{\tau_{b}} \simeq$ $100 \mathrm{TeV}$. Then according to (4.6), the gravity mediated gravitino mass has to be of the order $M_{3 / 2} \simeq 10^{5} \mathrm{TeV}$. Using (4.3), this leads to $F_{X} \simeq 10^{22} \mathrm{GeV}^{2}$. For gauge mediated soft masses of the order $500 \mathrm{GeV}$, we therefore get $x \simeq 5 \cdot 10^{16} \mathrm{GeV}$, which is slightly beyond the stronger limit (4.4). For further suppression of the sfermion masses there is no problem.

- In the first case one could ameliorate this problem by allowing for a certain tuning of the Higgs mass, so that the supersymmetry breaking scale for the visible sector can be larger than $500 \mathrm{GeV}$. Let us still have $F_{X} \simeq 10^{22} \mathrm{GeV}^{2}$ to avoid the CMP and require $x \simeq 5 \cdot 10^{14} \mathrm{GeV}$ to satisfy the constraint (4.4) for gauge mediation dominance. Then the gauge mediated soft masses are of the order $50 \mathrm{TeV}$.

Finally, let us discuss in which way this simple model of gauge mediation needs to be improved in order to show that it can really be embedded into string theory. As we already mentioned, we did not dynamically explain where the SUSY breaking field $X$ gets its VEV from. Recently, various kinds of models have been suggested, which, we think, so far are not completely convincing from a string theory point of view. One promising model is the so-called Fayet-Polonyi model. It combines an anomalous Peccei-Quinn symmetry with a linear superpotential in $X$ generated by another D3-instanton wrapping a del Pezzo surface of size $T_{\mathrm{FP}}$. This gives rise both to a D-term potential with a $T_{\mathrm{FP}}$ dependent FayetIliopoulos term and an F-term potential form the linear superpotential. Note, that the latter also depends on $T_{\mathrm{FP}}$. Now, also taking the Kähler potentials into account one has to show that dynamically really supersymmetry can be broken in such a way that the desired values for $x$ and $F_{X}$ arise. ${ }^{3}$ Moreover, one expects that also $F_{T_{\mathrm{FP}}} \neq 0$, which gives another source of supersymmetry breaking. Finally, one has to ensure that the moduli stabilisation in the bulk, i. e. of the $\tau_{b}$ and $\tau_{s}$ moduli and the moduli stabilisation of the local $X$ and $T_{\mathrm{FP}}$ moduli decouple. All these challenging questions are beyond the scope of this paper.

\subsection{Implications for the cosmological moduli problem}

Let us finish this section with some comments about the cosmological moduli problem [3941]. The cosmological moduli problem refers to the existence of late decaying moduli, with mass comparable to the gravitino. The moduli are expected to be displaced from their minimum during the inflationary epoch, subsequently oscillating about their minimum

\footnotetext{
${ }^{3}$ It was shown in [37], that this model with a simple choice of the Kähler potential actually still posses supersymmetric minima.
} 
and red-shifting as matter. The lifetime of such moduli is $\tau \sim \frac{M_{P}^{2}}{m_{\phi}^{3}} \gg 1 \mathrm{~s}$ for $m_{\phi} \lesssim 1 \mathrm{TeV}$. Moduli come to dominate the energy density of the universe, but if they decay too late then they fail to reheat the universe to temperatures sufficient for nucleosynthesis. In some ways the moduli problem is the most severe problem facing low-energy supersymmetry as it is very difficult to construct a viable cosmology with such long-lived moduli.

There are various possible approaches to this problem. In the absence of moduli-fixing mechanisms, it may have been hoped that one could stabilise the moduli at scales far above the gravitino mass. The more that has been learned about moduli stabilisation the less plausible this scenario has become.

The results in this paper suggest a novel approach to this problem. One of the properties of local LARGE volume GUTs with D-term stabilisation is that the soft terms appear at a scale hierarchically smaller than the gravitino mass. Depending on the extent of cancellations, we have seen that soft terms appear at an order not larger than $m_{\text {soft }} \sim \frac{M_{3 / 2}^{3 / 2}}{M_{P}^{1 / 2}}$, in the case when the dilaton F-term is responsible for uplifting. In all other cases gaugino masses will be further suppressed, with at least an extra loop factor as in anomaly mediation, and possibly even as far as $m_{\text {soft }} \sim \frac{M_{3 / 2}^{2}}{M_{P}}$. For the two extreme cases the gravitino mass appropriate to $\mathrm{TeV}$ soft terms is

$$
m_{\mathrm{soft}} \sim \frac{M_{3 / 2}^{3 / 2}}{M_{P}^{1 / 2}} \longrightarrow M_{3 / 2} \sim 10^{8} \mathrm{GeV} \quad m_{\mathrm{soft}} \sim \frac{M_{3 / 2}^{2}}{M_{P}} \longrightarrow M_{3 / 2} \sim 10^{11} \mathrm{GeV} .
$$

Instead of solving the moduli problem by making the moduli heavy and keeping soft terms comparable to the gravitino mass, this suggests making the gravitino heavy and having soft terms much lighter than the gravitino mass.

In the LARGE volume models the volume modulus $T_{b}$ is relatively light and has a mass $m_{T_{b}} \sim \frac{M_{3 / 2}^{3 / 2}}{M_{P}^{1 / 2}}$, while all other moduli have masses comparable to $M_{3 / 2}$. In the first case listed above, with a gravitino mass of around $10^{8} \mathrm{GeV}$, the volume modulus has $m \sim 1 \mathrm{TeV}$ and still poses cosmological problems. However in the other cases $m_{T_{b}}$ is sufficiently large to decay before nucleosynthesis. In the case of maximal suppression, with $M_{3 / 2} \sim 10^{11} \mathrm{GeV}$, then we have $m_{T_{b}} \sim 10^{7} \mathrm{GeV}$ with no cosmological problems. In all cases the other moduli (for example dilaton and complex structure moduli) have masses comparable to the gravitino mass and decay very rapidly.

It would also be interesting to study whether these suppressed soft terms would affect the thermal behaviour of the LARGE volume models studied in [42].

\section{Conclusions}

In this paper we have studied the structure of gravity mediated soft terms that arise when combining LARGE volume moduli stabilisation with local GUTs and D-term stabilisation of the cycle supporting the GUT brane.

We find that the modulus determining the size of the standard model cycle does not break supersymmetry and therefore the scale of gravity mediated soft terms is highly 
suppressed compared to the gravitino mass. Both "standard" gravity mediated terms of $\mathcal{O}\left(M_{3 / 2}\right)$ and also known anomaly mediated terms of $\mathcal{O}\left(g^{2} M_{3 / 2} / 16 \pi^{2}\right)$ vanish. The first non-zero terms appear to arise at $\mathcal{O}\left(\frac{M_{3 / 2}}{\sqrt{\mathcal{V}}}\right) \simeq \frac{M_{3 / 2}^{3 / 2}}{M_{P}^{1 / 2}}$. However it is possible that additional cancellations occur and suppress the soft terms even further than this down to $\mathcal{O}\left(\frac{M_{3 / 2}^{2}}{M_{P}}\right)$. The appearance of these further cancellations is related to the (in)dependence of the physical Yukawa couplings on the fields breaking supersymmetry.

The cancellation of contributions to the soft masses of order $M_{3 / 2}$ introduces several subtleties. In particular, as the soft terms occur at a scale parametrically smaller than the gravitino mass effects which are normally negligible become important. We have tried to include all known effects and have given general arguments as to when cancellations will take place. Nonetheless, it is important to look for any further possible contributions to soft terms which could possibly be dangerous. In this respect one would ideally like a direct stringy computation of soft terms that would bypass the need to go through the supergravity formalism.

The suppression of soft terms relative to the gravitino mass opens new avenues for thinking about the cosmological moduli problem. Rather than the traditional approach of making the moduli heavy while keeping the gravitino and soft terms around a $\mathrm{TeV}$, this opens the possibility of having the moduli and gravitino much heavier than a $\mathrm{TeV}$ while still maintaining TeVscale soft terms.

If the gravitational soft terms are of the order $\frac{M_{3 / 2}^{3 / 2}}{M_{P}^{1 / 2}}$, the volume modulus however remains a problem in the LARGE volume scenario as its mass is much lighter than the gravitino mass and would be the same order as the soft terms. If further cancellations occur and the soft terms are of order $\frac{M_{3 / 2}^{2}}{M_{P}}$, then the volume modulus ceases to be a cosmological problem.

Several scenarios regarding gravity and anomaly mediation are possible and which of these is actually realised may be model dependent. The main possibilities are:

- If the F-term of the dilaton field is responsible for the uplifting to de Sitter space, then $F^{S} \sim \mathcal{V}^{-3 / 2}$ and all the soft masses are of order $\frac{M_{P}}{\mathcal{V}^{3 / 2}} \sim \frac{M_{3 / 2}}{\sqrt{\mathcal{V}}}$. This is of the same order as the mass of the lightest modulus, the volume modulus, and this field remains dangerous for the cosmological moduli problem.

- If any other field is responsible for the de Sitter uplifting, the dilaton induces gravity mediated gaugino masses of order $\frac{M_{P}}{\mathcal{V}^{2}}$ or from anomaly mediation, barring any further cancellation, of order $\alpha \frac{M_{P}}{\mathcal{V}^{3 / 2}}$ where $\alpha$ is a loop factor. In both of these cases, identifying the gaugino masses with the $\mathrm{TeV}$ scale, the cosmological moduli problem is absent since the volume modulus would be at least as heavy as $10 \mathrm{TeV}$.

- For each of the two cases of the previous item, gravity mediated scalar masses, if not tachyonic, are of order $\frac{M_{P}}{\mathcal{V}^{3 / 2}}$ and therefore hierarchically heavier than the gaugino masses, indicating a minor version of split supersymmetry [43-45]. However if we have perfect sequestering in the sense that physical Yukawa couplings do not depend on 
the Kähler moduli fields that break supersymmetry, such terms will cancel. However scalar masses will always receive a contribution from the dilaton F-term at order $\frac{M_{P}}{\mathcal{V}^{2}}$.

- Since leading order gravity and anomaly mediation contributions to the soft terms are suppressed, then other effects have to be considered. In particular string loop corrections could be relevant, e.g. as in [46, 47], (giving potential contributions to scalar masses of order $\left.\frac{M_{P}}{\mathcal{V}^{5 / 3}}[48,49]\right)$ but also a novel scenario may be conceived in which the main source of supersymmetry breaking for the observable sector is gauge mediation, however the gravitino mass remains very large and unlike previous models of gauge mediation, the LSP is no longer the gravitino but can be a more standard neutralino.

Even though there are several scenarios, we can still extract some general conclusions from this analysis. First, as emphasised in [50], the effects of the de Sitter uplifting play an important rôle on the soft breaking terms. This is unlike previous scenarios based on the LARGE volume in which they were negligible. Second, in all scenarios the gravitino mass is much heavier than the $\mathrm{TeV}$ scale $M_{3 / 2} \geq 10^{8} \mathrm{GeV}$ which relaxes the cosmological problems associated to low-energy supersymmetry. Generically (except in the case that the dilaton is responsible for uplifting) the lightest modulus is heavier than the soft terms and therefore cosmologically harmless also.

Finally we point out that even though there are several cancellations that reduce the value of the volume to have the $\mathrm{TeV}$ scale, there is a minimum value of the volume that can be extracted from this analysis. Namely, the universal source of gaugino masses due to the dilaton dependence of the gauge kinetic function, implies that the gaugino masses cannot be smaller than $\frac{M_{P}}{\mathcal{V}^{2}}$. The same limit appears for scalar masses for the case of perfect sequestering $\left(Z=e^{\mathcal{K} / 3}\right)$. This provides a bound for the size of the overall volume $\mathcal{V} \sim 10^{6}-10^{7}$ in string units which corresponds to a string scale of order $M_{s} \sim 10^{15} \mathrm{GeV}$. Combining this with the recent result [23] that in local models the GUT unification scale is given by $M_{\mathrm{GUT}} \sim M_{s} \mathcal{V}^{1 / 6}$ this gives a unification scale of the same order as the one expected for supersymmetric GUT models from LEP precision results of $M_{\mathrm{GUT}} \sim 10^{16} \mathrm{GeV}$. If this scenario is actually realised it would provide an example in which a string model addresses simultaneously the two positive properties of the MSSM, namely the full hierarchy problem, without tuning, and obtaining the preferred scale of gauge unification.

Furthermore, this value of the volume is of the order of magnitude preferred by models of inflation in order for the inflaton to give rise to density perturbations of the right amplitude, normalised by COBE. In particular a volume $\mathcal{V} \sim 10^{5}-10^{7}$ was needed to achieve Kähler moduli inflation [51]. It also ameliorates the gravitino mass problem pointed out in $[52,53]$.

We consider our results bring closer local string/F-theory models to honest-to-God string compactifications since we incorporate the main properties of such models regarding supersymmetry breaking and moduli stabilisation. Many questions remain open. Concrete examples where the cancellations illustrated here are realised, including an uplifting term, loop corrections, etc. are desirable. The presence of such sub-leading contributions to soft terms can be recast in the presence of corrections to the physical Yukawa couplings. 
Specifically, the scale of the soft terms can be related to the extent to which the (local) physical Yukawa couplings depend on the (bulk) supersymmetry breaking fields. In the limit of perfect sequestering the Kähler moduli contribution to soft masses vanish. It may be possible to study this issue more precisely using the techniques of orbifold CFT. Furthermore, for F-theory constructions, even though in general they are treated in a way similar to orientifold constructions, the $4 \mathrm{D}$ effective field theory for F-theory models is less under control. In particular the $\alpha^{\prime}$-corrections which are crucial in the large volume scenario, need to be computed for F-theory compactifications.

\section{Acknowledgments}

We gratefully acknowledge discussions with C.P. Burgess, B. Campbell, K. Choi, M. Cicoli, M. Dolan, T. Grimm, L. Ibáñez, D. Lüst, A. Maharana, F. Marchesano, E. Palti, E. Plauschinn, M. Schmidt-Sommerfeld, A. Uranga, G. Villadoro, T. Weigand and E. Witten. FQ wants to particularly thank S. de Alwis for many enlightning discussions on related subjects. RB would like to thank the Galileo Galilei Institute for Theoretical Physics for hospitality and the INFN for partial support during the completion of this work. SLK would like to thank the CERN Theory group for hospitality at the early stages of this project. JC is grateful to the Royal Society for a University Research Fellowship.

\section{A F-Terms}

As there is a cancellation at leading order taking place in the calculation of various soft terms, a careful large volume expansion up to the next-to-leading order has to be performed. Let us start with the expressions for $e^{-a \tau_{s}}$ and $\tau_{s}^{3 / 2}$ in the minimum, which will be needed later.

For this purpose, consider the scalar potential (2.13). Upon minimising it with respect to the two independent variables $\tau_{s}$ and $\mathcal{V}$, we get two expression: First, from the condition $\frac{\partial V_{F}}{\partial \tau_{s}}=0$, it follows:

$$
e^{-a \tau_{s}}=\frac{\mu}{\lambda} \frac{\left|W_{0}\right|}{a A \mathcal{V}} \frac{1}{\sqrt{\tau_{s}}} \frac{\left(1-a \tau_{s}\right)}{\left(-2 a+\frac{1}{2 \tau_{s}}\right)} .
$$

After developing the denominator in powers of $1 /\left(a \tau_{s}\right)$ and inserting the expressions for $\mu$ and $\lambda$ we get

$$
e^{-a \tau_{s}} \approx \frac{3}{4} \frac{\eta_{s}^{3 / 2}}{a A} \sqrt{\tau_{s}} \frac{W_{0}}{\mathcal{V}}\left(1-\frac{3}{4 a \tau_{s}}\right)
$$

The second expression arises upon solving $\frac{\partial V_{F}}{\partial \mathcal{V}}=0$ for $\tau_{s}^{3 / 2}$ and thereby using (A.2). The result is

$$
\tau_{s}^{3 / 2} \approx \frac{\hat{\xi}}{2 \eta_{s}^{3 / 2}}\left(1+\frac{1}{2 a \tau_{s}}\right) .
$$

Another approximation needed in the following is:

$$
\mathcal{K}^{a b}\left(\partial_{b} \mathcal{K}\right)=-\frac{4 \mathcal{V}^{2}+\mathcal{V} \hat{\xi}+4 \hat{\xi}^{2}}{2(\mathcal{V}-\hat{\xi})\left(\mathcal{V}+\frac{\hat{\xi}}{2}\right)} \tau_{a} \approx-2 \tau_{a}-\frac{3}{2} \hat{\xi} \frac{\tau_{a}}{\mathcal{V}}
$$


where the sum runs only over Kähler moduli. The first equality can be derived using the expressions for the Kähler metric and the derivatives of the Kähler potential with respect to the moduli in terms of two-cycle volumes $t^{a}$ instead of four-cycle volumes $\tau_{a}$ (see $[2,54]$ ). We are now in a position to calculate $F^{b}$ :

$$
F^{b}=e^{\mathcal{K} / 2} \mathcal{K}^{b J} D_{J} W=e^{\mathcal{K} / 2}\left(\mathcal{K}^{b \tau_{j}}\left(\partial_{\tau_{j}} \mathcal{K}\right) W+\mathcal{K}^{b s}\left(\partial_{s} W\right)+\mathcal{K}^{b S} D_{S} W\right)
$$

The term involving $D_{S} W$ turns out to be sub-leading in the $\mathcal{V}^{-1}$ expansion and can be neglected (see below). The derivative of the superpotential with respect to $T_{s}$ undergoes a sign-flip due to the minimisation with respect to the corresponding axion as argued in [2]. Using the approximations (A.2), (A.3) and (A.4), one easily gets:

$$
F^{b}=-2 \tau_{b} \frac{\sqrt{g_{s}}}{\sqrt{2}} \frac{W_{0}}{\mathcal{V}}-\frac{3}{8 \sqrt{2}} \frac{\tau_{b}}{a \tau_{s}}\left(1+\frac{3}{2 a \tau_{s}}\right) \frac{W_{0}}{\mathcal{V}^{2}}+\mathcal{O}\left(\mathcal{V}^{-3}\right)
$$

or with the expressions for the gravitino- and gaugino-mass (3.2) and (3.4) inserted:

$$
F^{b}=-2 \tau_{b} M_{3 / 2}-\frac{\tau_{b}}{2 a \tau_{s}}\left(1+\frac{3}{2 a \tau_{s}}\right) M_{\widetilde{G}}+\mathcal{O}\left(\mathcal{V}^{-3}\right) .
$$

From (A.2), it can be derived that $a \tau_{s} \approx \ln \mathcal{V} \approx 10$. Thus, for the sake of shorter formulae, one may also neglect the second term in the parenthesis:

$$
F^{b} \approx-2 \tau_{b} M_{3 / 2}-\frac{\tau_{b}}{2 a \tau_{s}} M_{\widetilde{G}}
$$

Next, we want to calculate $F^{S}=e^{\mathcal{K} / 2} K^{S \bar{J}} D_{\bar{J}} \bar{W}$. Here, a subtlety arises concerning $D_{S} W=\partial_{S} W+W\left(\partial_{S} \mathcal{K}\right)$ : the Kähler potential depends on the dilaton not only in the usual way via $-\ln (S+\bar{S})$, but there is also a contribution in the $\alpha^{\prime}$-correction in the Kähler moduli part. Thus, $\partial_{S} \mathcal{K}$ has $\mathcal{V}^{-1}$ corrections:

$$
D_{S} W \approx \partial_{S} W_{0}-\frac{g_{s}}{2} W_{0}-\frac{3}{4} \frac{\xi}{g_{s}^{1 / 2}} \frac{W_{0}}{\mathcal{V}}+\mathcal{O}\left(\mathcal{V}^{-2}\right)
$$

Also as a consequence of the $\alpha^{\prime}$-corrections, the minimum of the scalar potential for the dilaton is shifted away from the supersymmetric locus $D_{S} W=0$ at order $\mathcal{V}^{-1}$. In order to determine the new minimum, one would have to minimise the full potential, before integrating out the dilaton. However, since we do not have an explicit model with a full flux sector, in order to capture this effect, we assume that the two leading order terms in (A.9) cancel and keep only the next-to-leading order terms in the $\mathcal{V}^{-1}$ expansion. The expression we get in this way has certainly the correct order in $\mathcal{V}$ and we include an order one constant $\gamma$ in the final result comprising the uncertainty about the true location of the new minimum.

$$
D_{S} W \approx-\frac{3}{4} \gamma^{\prime} \frac{\xi}{g_{s}^{1 / 2}} \frac{W_{0}}{\mathcal{V}}
$$

In the sum over $D_{I} W$ in the dilaton $\mathrm{F}$-Term $F^{S}=e^{\mathcal{K} / 2} \mathcal{K}^{S J} D_{J} W$, there are finally two contributions at order $\mathcal{V}^{-2}$ : one from $\mathcal{K}^{S b} F_{b}$ and one from $\mathcal{K}^{S S} F_{S}$. The result reads:

$$
F^{S} \approx \frac{3}{2 \sqrt{2}} \gamma \frac{\xi}{g_{s}^{2}} \frac{W_{0}}{\mathcal{V}^{2}}
$$




\section{B Vanishing D-terms including matter}

We will consider in this appendix a concrete example with a generic D-term including not only the field dependent FI-term but also a charged matter field. In general vanishing Dterms do not imply vanishing FI-term but a cancellation between the two terms entering the D-term potential. We argue here (following [55]) that once soft supersymmetry breaking terms are included, as long as the square of the scalar masses is positive the minimum of the scalar potential is for vanishing both matter field VEV and FI-term.

Since in local models the standard model cycle is a del Pezzo surface that can and usually prefers to shrink to small size, it is dangerous to work in the regime where the cycle size is larger than the string scale. Even though at sizes of the order of the string scale the spectrum and couplings of the model are not understood, the regime close to a del Pezzo singularity is under a much better control, the spectrum is determined by the extended quiver diagrams and the low-energy effective theory can be reliably used in an expansion in the small blow-up mode.

This effective field theory has been recently discussed in [9]. We start with the same background geometry as before including one large $\tau_{1}$ and two small cycles $\tau_{2}$, $\tau_{3}$. On the rigid cycle $\tau_{2}$ we have the standard non-perturbative effect. Being at the singular locus for $\tau_{3}$, the effective field theory can be approximated by the following supergravity set-up:

$$
\begin{aligned}
\mathcal{K} & =-2 \log \left(\mathcal{V}+\frac{\hat{\xi}}{2}\right)+\frac{\alpha \tau_{3}^{2}}{\mathcal{V}}+Z|\varphi|^{2} \\
W & =W_{0}+A e^{-a T_{2}} \\
f & =d T_{3}+S
\end{aligned}
$$

where $\varphi$ denotes a matter field that is charged under an anomalous $\mathrm{U}(1)$ on the standard model cycle, as is the cycle volume itself. As discussed in [9], the effective theory for $\tau_{3}$ differs from the standard treatment for relatively large values of $\tau_{3}$ since we are working close to the singularity. The anomalous U(1) generates a D-term potential with a FayetIliopoulos term:

$$
V_{D}=\frac{1}{2\left(d \tau_{3}+s\right)}\left(Q_{\varphi} Z|\varphi|^{2}+\frac{Q_{\tau_{3}} \tau_{3}}{\mathcal{V}}\right)^{2}
$$

The matter metric $Z$ is taken to have the general form

$$
Z=\frac{1}{\mathcal{V}^{2 / 3}}\left(\beta+\gamma \tau_{3}^{\lambda}-\frac{\delta}{\mathcal{V}}\right)
$$

where the constants $\beta, \delta$ can in principle depend on the dilaton and complex structure moduli.

The D-term potential determines the size of $\tau_{3}$ and implies

$$
\tau_{3} \sim|\varphi|^{2} \mathcal{V}^{1 / 3}
$$

For a vanishing VEV of $\varphi$ this implies as previously $\tau_{3}=0$. Expanding around $\varphi=0$, the scalar potential is given by the standard LARGE volume potential and at next-to-leading 
order by a contribution quadratic in $\varphi$ :

$$
\begin{aligned}
V= & \frac{1}{\left(\mathcal{V}+\frac{\xi}{2}\right)^{2}}\left(\frac{8}{3}|a A|^{2} \sqrt{\tau_{2}} \tau_{1}^{3 / 2} e^{-2 a \tau_{2}}-4 W_{0} a A \tau_{2} e^{-a \tau_{2}}+\frac{W_{0}^{2} 3 \xi}{4 \tau_{1}^{3 / 2}}+Y\right. \\
& \left.-\frac{\beta|\varphi|^{2}}{3 \tau_{1}}\left(\frac{8}{3}|a A|^{2} \sqrt{\tau_{2}} \tau_{1}^{3 / 2} e^{-2 a \tau_{2}}-4 W_{0} a A \tau_{2} e^{-a \tau_{2}}+\frac{9 W_{0}^{2}\left(5 \frac{\delta}{\beta}+2 \xi\right)}{4 \tau_{1}^{3 / 2}}\right)\right) \\
& +\frac{\beta|\varphi|^{2}}{\tau_{1}\left(\mathcal{V}+\frac{\xi}{2}\right)^{2}}\left(\frac{8}{3}|a A|^{2} \sqrt{\tau_{2}} \tau_{1}^{3 / 2} e^{-2 a \tau_{2}}-4 W_{0} a A \tau_{2} e^{-a \tau_{2}}+\frac{3 W_{0}^{2} \xi}{4 \tau_{1}^{3 / 2}}+Y\right),
\end{aligned}
$$

where the last term arises from the expansion of $e^{\mathcal{K}}$ and $Y$ denotes the F-term uplifting term, which allows for a stabilisation at zero vacuum energy.

With zero vacuum energy, the mass of $\varphi$ is given by

$$
\begin{aligned}
m_{\varphi}^{2} & =\mathcal{K}_{\varphi \varphi}^{-1} \frac{-\beta}{3 \tau_{1}^{4}}\left(\frac{8}{3}|a A|^{2} \sqrt{\tau_{2}} \tau_{1}^{3 / 2} e^{-2 a \tau_{2}}-4 W_{0} a A \tau_{2} e^{-a \tau_{2}}+\frac{9 W_{0}^{2}\left(2 \xi-5 \frac{\delta}{\beta}\right)}{4 \tau_{1}^{3 / 2}}\right) \\
& =-\frac{1}{3 \tau_{1}^{3}}\left(V_{\min }+\frac{45 W_{0}^{2}\left(\frac{\xi}{3}-\frac{\delta}{\beta}\right)}{4 \tau_{1}^{3 / 2}}\right) \approx \frac{15 W_{0}^{2}\left(\frac{\delta}{\beta}-\frac{\xi}{3}\right)}{4 \tau_{1}^{9 / 2}}
\end{aligned}
$$

Different ratios of $\delta / \beta$ allow for tachyonic, zero or positive masses at this order. In particular:

$$
\frac{\delta}{\beta} \begin{cases}<\frac{\xi}{3} & \text { tachyonic } \\ =\frac{\xi}{3} & \text { zero } \\ >\frac{\xi}{3} & \text { positive. }\end{cases}
$$

With respect to the matter metric the condition $\frac{\delta}{\beta}=\frac{\xi}{3}$ can be understood as follows: The case of vanishing masses corresponds to the following matter metric:

$$
Z=\frac{\beta}{\mathcal{V}^{2 / 3}}\left(1-\frac{\xi}{3 \mathcal{V}}\right) \approx \frac{\beta}{\left(\mathcal{V}+\frac{\xi}{2}\right)^{2 / 3}}=\beta e^{\mathcal{K} / 3},
$$

which is the condition found in section 3.3 for extreme sequestering and cancellation of scalar masses at the $1 / \mathcal{V}^{3 / 2}$ level. Without the uplifting term, the effect of the term arising from the expansion of $e^{\mathcal{K}}$ is generally sub-leading to the other contribution since it is suppressed with $1 / a \tau_{2}$.

For positive scalar masses we can clearly see that combining the term $m_{\varphi}^{2} \varphi^{2}$ with the D-term potential, both the VEV of $\varphi$ and the FI-term vanish at the minimum as desired. For the tachyonic case this would indicate as usual that at the minimum the scalar field and the FI-term would be non-vanishing. If $\varphi$ is a field charged under the standard model gauge group this is undesirable since it would break the standard model symmetries at high energies. If the condition $\frac{\delta}{\beta}=\frac{\xi}{3}$ is satisfied the positivity of the squared scalar masses at a higher order would have to be determined. 


\section{References}

[1] S. Kachru, R. Kallosh, A. Linde and S.P. Trivedi, De Sitter vacua in string theory, Phys. Rev. D 68 (2003) 046005 [hep-th/0301240] [SPIRES].

[2] V. Balasubramanian, P. Berglund, J.P. Conlon and F. Quevedo, Systematics of moduli stabilisation in Calabi-Yau flux compactifications, JHEP 03 (2005) 007 [hep-th/0502058] [SPIRES].

[3] J.P. Conlon, F. Quevedo and K. Suruliz, Large-volume flux compactifications: moduli spectrum and D3/D7 soft supersymmetry breaking, JHEP 08 (2005) 007 [hep-th/0505076] [SPIRES].

[4] M. Cicoli, J.P. Conlon and F. Quevedo, General analysis of LARGE volume scenarios with string loop moduli stabilisation, JHEP 10 (2008) 105 [arXiv:0805.1029] [SPIRES].

[5] J.P. Conlon, S.S. AbdusSalam, F. Quevedo and K. Suruliz, Soft SUSY breaking terms for chiral matter in IIB string compactifications, JHEP 01 (2007) 032 [hep-th/0610129] [SPIRES].

[6] J.P. Conlon, C.H. Kom, K. Suruliz, B.C. Allanach and F. Quevedo, Sparticle spectra and LHC signatures for large volume string compactifications, JHEP 08 (2007) 061 [arXiv:0704.3403] [SPIRES].

[7] R. Blumenhagen, S. Moster and E. Plauschinn, Moduli stabilisation versus chirality for MSSM like type IIB orientifolds, JHEP 01 (2008) 058 [arXiv:0711.3389] [SPIRES].

[8] G. Aldazabal, L.E. Ibáñez, F. Quevedo and A.M. Uranga, D-branes at singularities: a bottom-up approach to the string embedding of the standard model, JHEP 08 (2000) 002 [hep-th/0005067] [SPIRES].

[9] J.P. Conlon, A. Maharana and F. Quevedo, Towards realistic string vacua, JHEP 05 (2009) 109 [arXiv:0810.5660] [SPIRES].

[10] R. Blumenhagen, B. Körs, D. Lüst and T. Ott, The standard model from stable intersecting brane world orbifolds, Nucl. Phys. B 616 (2001) 3 [hep-th/0107138] [SPIRES].

[11] R. Donagi and M. Wijnholt, Model building with F-theory, arXiv:0802.2969 [SPIRES].

[12] C. Beasley, J.J. Heckman and C. Vafa, GUTs and exceptional branes in F-theory - I, JHEP 01 (2009) 058 [arXiv:0802.3391] [SPIRES].

[13] C. Beasley, J.J. Heckman and C. Vafa, GUTs and exceptional branes in F-theory - II: experimental predictions, JHEP 01 (2009) 059 [arXiv:0806.0102] [SPIRES].

[14] R. Donagi and M. Wijnholt, Breaking GUT groups in F-theory, arXiv:0808.2223 [SPIRES].

[15] R. Donagi and M. Wijnholt, Higgs bundles and UV completion in F-theory, arXiv:0904.1218 [SPIRES].

[16] J. Marsano, N. Saulina and S. Schäfer-Nameki, F-theory compactifications for supersymmetric GUTs, JHEP 08 (2009) 030 [arXiv:0904.3932] [SPIRES].

[17] A. Collinucci, New F-theory lifts II: permutation orientifolds and enhanced singularities, arXiv:0906.0003 [SPIRES].

[18] R. Blumenhagen, T.W. Grimm, B. Jurke and T. Weigand, F-theory uplifts and GUTs, arXiv:0906.0013 [SPIRES].

[19] J.J. Heckman, J. Marsano, N. Saulina, S. Schäfer-Nameki and C. Vafa, Instantons and SUSY breaking in F-theory, arXiv:0808.1286 [SPIRES]. 
[20] J.J. Heckman and C. Vafa, F-theory, GUTs and the weak scale, arXiv:0809.1098 [SPIRES].

[21] R. Blumenhagen, V. Braun, T.W. Grimm and T. Weigand, GUTs in type IIB orientifold compactifications, Nucl. Phys. B $\mathbf{8 1 5}$ (2009) 1 [arXiv:0811.2936] [SPIRES].

[22] R. Blumenhagen, Gauge coupling unification in F-theory grand unified theories, Phys. Rev. Lett. 102 (2009) 071601 [arXiv:0812.0248] [SPIRES].

[23] J.P. Conlon, Gauge threshold corrections for local string models, JHEP 04 (2009) 059 [arXiv:0901.4350] [SPIRES].

[24] J.P. Conlon and E. Palti, Gauge threshold corrections for local orientifolds, arXiv:0906.1920 [SPIRES].

[25] J.P. Conlon and E. Palti, On gauge threshold corrections for local IIB/F-theory GUTs, arXiv:0907.1362 [SPIRES].

[26] S. Gukov, C. Vafa and E. Witten, CFT's from Calabi-Yau four-folds, Nucl. Phys. B 584 (2000) 69 [hep-th/9906070] [SPIRES].

[27] K. Becker, M. Becker, M. Haack and J. Louis, Supersymmetry breaking and $\alpha^{\prime}$-corrections to flux induced potentials, JHEP 06 (2002) 060 [hep-th/0204254] [SPIRES].

[28] S.P. de Alwis, On integrating out heavy fields in SUSY theories, Phys. Lett. B 628 (2005) 183 [hep-th/0506267] [SPIRES].

[29] L. Brizi, M. Gomez-Reino and C.A. Scrucca, Globally and locally supersymmetric effective theories for light fields, Nucl. Phys. B 820 (2009) 193 [arXiv:0904.0370] [SPIRES].

[30] A. Achucarro, S. Hardeman and K. Sousa, Consistent decoupling of heavy scalars and moduli in $N=1$ supergravity, Phys. Rev. D 78 (2008) 101901 [arXiv:0806.4364] [SPIRES].

[31] D. Gallego and M. Serone, An effective description of the landscape - I, JHEP 01 (2009) 056 [arXiv:0812.0369] [SPIRES].

[32] J.P. Conlon, D. Cremades and F. Quevedo, Kähler potentials of chiral matter fields for Calabi-Yau string compactifications, JHEP 01 (2007) 022 [hep-th/0609180] [SPIRES].

[33] L. Aparicio, D.G. Cerdeno and L.E. Ibáñez, Modulus-dominated SUSY-breaking soft terms in F-theory and their test at LHC, JHEP 07 (2008) 099 [arXiv:0805.2943] [SPIRES].

[34] J.A. Bagger, T. Moroi and E. Poppitz, Anomaly mediation in supergravity theories, JHEP 04 (2000) 009 [hep-th/9911029] [SPIRES].

[35] S.P. de Alwis, On anomaly mediated SUSY breaking, Phys. Rev. D 77 (2008) 105020 [arXiv: 0801.0578] [SPIRES].

[36] M. Cvetič and T. Weigand, A string theoretic model of gauge mediated supersymmetry beaking, arXiv:0807.3953 [SPIRES].

[37] E. Dudas, Y. Mambrini, S. Pokorski, A. Romagnoni and M. Trapletti, Gauge vs. gravity mediation in models with anomalous $\mathrm{U}(1)$ 's, JHEP 03 (2009) 011 [arXiv:0809.5064] [SPIRES].

[38] D. Green and T. Weigand, Retrofitting and the $\mu$ problem, arXiv:0906.0595 [SPIRES].

[39] G.D. Coughlan, W. Fischler, E.W. Kolb, S. Raby and G.G. Ross, Cosmological problems for the Polonyi potential, Phys. Lett. B 131 (1983) 59 [SPIRES].

[40] B. de Carlos, J.A. Casas, F. Quevedo and E. Roulet, Model independent properties and cosmological implications of the dilaton and moduli sectors of 4-D strings,

Phys. Lett. B 318 (1993) 447 [hep-ph/9308325] [SPIRES]. 
[41] T. Banks, D.B. Kaplan and A.E. Nelson, Cosmological implications of dynamical supersymmetry breaking, Phys. Rev. D 49 (1994) 779 [hep-ph/9308292] [SPIRES].

[42] L. Anguelova, V. Calo and M. Cicoli, LARGE volume string compactifications at finite temperature, arXiv:0904.0051 [SPIRES].

[43] J.D. Wells, Implications of supersymmetry breaking with a little hierarchy between gauginos and scalars, hep-ph/0306127 [SPIRES].

[44] J.D. Wells, PeV-scale supersymmetry, Phys. Rev. D 71 (2005) 015013 [hep-ph/0411041] [SPIRES].

[45] N. Arkani-Hamed and S. Dimopoulos, Supersymmetric unification without low energy supersymmetry and signatures for fine-tuning at the LHC, JHEP 06 (2005) 073 [hep-th/0405159] [SPIRES].

[46] M. Berg, M. Haack and B. Körs, String loop corrections to Kähler potentials in orientifolds, JHEP 11 (2005) 030 [hep-th/0508043] [SPIRES].

[47] M. Berg, M. Haack and E. Pajer, Jumping through loops: on soft terms from large volume compactifications, JHEP 09 (2007) 031 [arXiv:0704.0737] [SPIRES].

[48] S.P. de Alwis, Mediation of supersymmetry breaking in a class of string theory models, JHEP 03 (2009) 023 [arXiv:0806.2672] [SPIRES].

[49] S.P. de Alwis et. al., to appear.

[50] S.P. de Alwis, String phenomenology and the cosmological constant, Phys. Lett. B 647 (2007) 194 [hep-th/0607148] [SPIRES].

[51] J.P. Conlon and F. Quevedo, Kähler moduli inflation, JHEP 01 (2006) 146 [hep-th/0509012] [SPIRES].

[52] R. Kallosh and A. Linde, Landscape, the scale of SUSY breaking and inflation, JHEP 12 (2004) 004 [hep-th/0411011] [SPIRES].

[53] J.P. Conlon, R. Kallosh, A. Linde and F. Quevedo, Volume modulus inflation and the gravitino mass problem, JCAP 09 (2008) 011 [arXiv:0806.0809] [SPIRES].

[54] K. Bobkov, Volume stabilization via $\alpha^{\prime}$ corrections in type IIB theory with fluxes, JHEP 05 (2005) 010 [hep-th/0412239] [SPIRES].

[55] J.F.G. Cascales, M.P. Garcia del Moral, F. Quevedo and A.M. Uranga, Realistic D-brane models on warped throats: fluxes, hierarchies and moduli stabilization, JHEP 02 (2004) 031 [hep-th/0312051] [SPIRES]. 